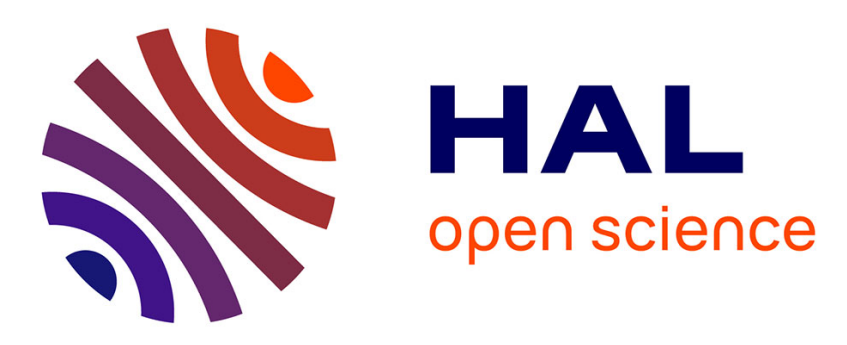

\title{
Impact of polyacrylamide adsorption on flow through porous siliceous materials: State of the art, discussion and industrial concern
}

\author{
H Bessaies-Bey, J Fusier, Simon Harrisson, Mathias Destarac, S Jouenne, N \\ Passade-Boupat, F Lequeux, J.-B. d'Espinose de Lacaillerie, N Sanson
}

\section{To cite this version:}

H Bessaies-Bey, J Fusier, Simon Harrisson, Mathias Destarac, S Jouenne, et al.. Impact of polyacrylamide adsorption on flow through porous siliceous materials: State of the art, discussion and industrial concern. Journal of Colloid and Interface Science, 2018, 531, pp.693-704. 10.1016/j.jcis.2018.07.103 . hal-01894835

\section{HAL Id: hal-01894835 \\ https://hal.science/hal-01894835}

Submitted on 12 Oct 2018

HAL is a multi-disciplinary open access archive for the deposit and dissemination of scientific research documents, whether they are published or not. The documents may come from teaching and research institutions in France or abroad, or from public or private research centers.
L'archive ouverte pluridisciplinaire HAL, est destinée au dépôt et à la diffusion de documents scientifiques de niveau recherche, publiés ou non, émanant des établissements d'enseignement et de recherche français ou étrangers, des laboratoires publics ou privés. 


\section{Impact of Polyacrylamide Adsorption on Flow through Porous Siliceous Materials: State of the Art, Discussion and Industrial Concern}

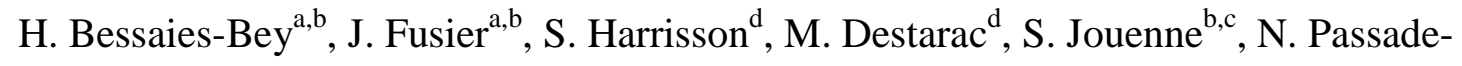
Boupat $^{\text {b,c }}$, F. Lequeux ${ }^{\mathrm{a}, \mathrm{b}}$, J.-B. d'Espinose de Lacaillerie ${ }^{\mathrm{a}, \mathrm{b}}$, N. Sanson ${ }^{*}, \mathrm{a}, \mathrm{b}$

${ }^{\text {a } S o f t ~ M a t t e r ~ S c i e n c e ~ a n d ~ E n g i n e e r i n g ~ l a b o r a t o r y, ~ U M R ~ C N R S ~ 7615, ~ E S P C I ~ P a r i s, ~ P S L ~}$ University, Sorbonne Université, F-75005 Paris.

${ }^{\mathrm{b}}$ Laboratoire Physico-chimie des Interfaces Complexes, ESPCI Paris, 10 rue Vauquelin, F75231 Paris, Route Départementale 817, 64170 Lacq, France.

${ }^{\mathrm{c}}$ TOTAL CSTJF, Avenue Larribau, Pau, France.

${ }^{\mathrm{d}}$ Université Paul Sabatier, Laboratoire des Interactions Moléculaires et de la Réactivité Chimique et Photochimique, UMR-CNRS 5623, Bât 2R1, 118 route de Narbonne, 31062 Toulouse, France. 
* Corresponding author.

E-mail address: nicolas.sanson@espci.fr (N. Sanson)

Present address: Soft Matter Science and Engineering laboratory, UMR CNRS 7615, ESPCI

Paris, PSL University, Sorbonne Université, F-75005 Paris. Laboratoire Physico-chimie des

Interfaces Complexes, ESPCI Paris, 10 rue Vauquelin, F-75231 Paris, Route Départementale 817, 64170 Lacq, France.

Phone: +33140794 417

Keywords:

Silica

Polyacrylamide (PAM/HPAM)

Adsorption

Flow

Injectivity 


\begin{abstract}
Hypothesis

Adsorption of high molecular mass polymers impacts flow in porous media. In the industrially crucial case of acrylamide-based polymers in porous silicates, the very occurrence of adsorption is still debated. Thus, the present work aimed at establishing a clear correlation between adsorption of acrylamide-based polymers and injectivity loss in porous silica.

Experiments

A review of the literature revealed apparent discrepancies regarding the affinity of acrylamide-based polymers for siliceous materials having ostensibly the same chemical composition. Through a deeper analysis of the reported literature and new experimental measurements on well-defined polymers and surfaces, we investigated the relation between the silica surface properties and the acrylamide-based polymer adsorption. Our observations were confronted with water injection experiments in porous media of different surface compositions previously put in contact with polymers.

\section{Findings}

The polymer affinity towards the silica surface depended on the density of hydroxyl groups at the surface of the oxide, its thermal treatment, storage condition and purity. This demonstrated that the impact of adsorption on acrylamide-based polymer flow within porous silicates heavily depends on the silicate surface composition and must be carefully evaluated. In view of the continually expanding use of acrylamide-based polymers, notably in enhanced oil recovery, such considerations provide interesting insights into the effect of adsorption on their flow into porous materials.
\end{abstract}




\section{Introduction}

The flow of high molar mass polymers in porous media is particularly complex. The combination of distributed pore geometry, non-newtonian rheological properties in confined geometry, the extensional character of the flow and the adsorption of polymer chains on the pore wall, all lead to a behavior which is difficult to predict beforehand. Nevertheless, considerable efforts have been deployed in that direction because of the industrial importance of the issue. This is particularly true in the field of enhanced oil recovery (EOR), where highmolar mass water-soluble polymers are used as mobility control agents. In this context, G. Chauveteau already identified and reviewed thirty years ago the underlying physics governing polymer flow in porous media and since then, through continuous studies, a relatively good understanding has been reached. ${ }^{1}$ In particular, the importance of adsorption on polymer retention and flow has been underlined..$^{2-4}$ Remarkably, the impact of adsorption on flow can be sometimes counterintuitive, combining effects of opposite consequences such as surface depletion (which can reduce or favor retention depending on the permeability of the adsorbed layer) and hydrodynamic radii reduction (which varies non monotonously with the flow rate). Disentangling the impact of these complex physical effects in real systems is only possible if the chemistry governing polymer adsorption is correctly described. For that purpose, the specificity of each system must be examined carefully, i. e. the structure of the polymer under consideration, the solution chemistry (ionic strength, $\mathrm{pH}$, temperature, ...) and the nature of the porous solid through which the flow takes place. When considering the flow of acrylamide-based polymers within porous oxides, one can benefit from a large pre-existing literature dedicated to their adsorption on various model oxides. This is a mixed blessing. However, because a multiplicity of parameters impact adsorption, this literature can appear sometimes confusing in its diversity of systems and objectives. The goal of this paper is thus to clarify the role of the specific interaction of acrylamide-based polymers with siliceous materials on adsorption and its impact on flow resistance.

Acrylamide-based polymers such as neutral polyacrylamide (PAM) or partially hydrolyzed polyacrylamide (HPAM) are employed in a wide range of industrial applications where a good knowledge of polymer behavior at solid-liquid interfaces plays a major role. Many industrial processes such as biotechnology, water treatment, and cosmetics involve polyacrylamide in order to tailor the colloidal state of particles. For instance, acrylamidebased polymers are mainly used to enhance soil stability and cohesion through flocculation of 
colloidal particles. ${ }^{5-15}$ They are also deployed in the construction materials industry in order to tailor the rheology of these materials at the fresh state during the casting process. ${ }^{16-20}$ As already stated, another important example can be found in EOR, where PAM/HPAM are used as mobility control agents. In this case, the adsorption of polymer onto mineral surfaces leads to an erosion of the viscous front and affect the injectivity process. ${ }^{21}$ In all these examples, polymers are put into contact with minerals having different surface properties and compositions which means that the polymer affinity with these surfaces is one of the most fundamental processes involved in their mechanism of action. In order to understand the nature and strength of the polymer adsorption process onto the particle surface, researchers have employed pure oxides as model systems such as silica, alumina, or hematite. The adsorption of the polymers onto the particle surface depends not only on the affinity of the polymer for the surface and consequently of the chemical nature of both the polymer repeat units and the surface groups of the substrate but also of the physicochemical parameters such as $\mathrm{pH}$, temperature and ionic strength. Consequently, there exists a large amount of literature dedicated to the study of the interactions between acrylamide-based polymers on the surface of oxides, notably siliceous materials. However, the reported results appear sometimes contradictory and there is no short answer on whether they do adsorb or not. ${ }^{22-38}$ In view of the importance of the question in many industrial processes, the objective of the present work is to rationalize the apparent differences observed in literature concerning acrylamide-based polymers affinity for siliceous materials having ostensibly the same chemical composition.

To shed light on this question: (i) as a preamble, we present the literature background on the specific issue of adsorption or lack thereof of acrylamide-based polymer on siliceous materials. We concurrently discuss first the parameters impacting both the silica surface and the physico-chemical behavior of the polymer in solution such as $\mathrm{pH}$, temperature and polymer chemical composition, and secondly, the state of the silica surface such as the nature of surface groups, the presence of impurity and the temperature treatment, ... (ii) Then, we complete the existing literature by adsorption experiments on well-characterized siliceous materials of acrylamide-based polymers synthesized by controlled radical polymerization leading to well-defined average molar masses, molar mass distributions and chemical compositions. (iii) Finally, we correlate the results obtained in this work with injectivity measurements of water on silica porous materials previously contacted with polymers. From this, we suggest that polymer adsorption might account in part for polymer injectivity losses through the build up of a resistance to flow during enhanced oil recovery. 


\section{Materials and methods}

\subsection{Materials}

Siliceous materials. Different types of siliceous adsorbent were used in this study: a crystallized natural quartz $\left(99.5 \% \mathrm{SiO}_{2}, 0.5 \% \mathrm{Al}_{2} \mathrm{O}_{3}\right)$, named Sikaisol and acquired commercially from Sika, Specific area $\mathrm{S}=0.56 \mathrm{~m}^{2} / \mathrm{g}$, determined by BET technique; bulk density $\rho=2650 \mathrm{~kg} / \mathrm{m}^{3}$; mean particle diameter $\mathrm{D}=50 \mu \mathrm{m}$ ); Synthetic amorphous silica particles from Fiber Optic Center (Specific area $S=1.1 \mathrm{~m}^{2} / \mathrm{g}$, bulk density $\rho=1800 \mathrm{~kg} / \mathrm{m}^{3}$, mean particle diameter $\mathrm{D}=1.5 \mu \mathrm{m}$ ); Siliceous porous filter discs from Heraeus (diameter of $25.4 \mathrm{~mm}$ and a thickness of $8 \mathrm{~mm}$, pore size 10-16 $\mu \mathrm{m}$ ) for injectivity experiments. The Aluminum treatment was realized by putting in contact the silica materials with a solution of $\mathrm{Al}_{13}$ polycations ${ }^{39}$ in agreement with Parneix et al.. ${ }^{40}$

Polymers. Acrylamide (AM) (40\% aqueous solution) and acrylic acid (AA) (99\%) were purchased from Sigma-Aldrich. Well-defined acrylamide-based polymers, PAM and P(AMst-AA) with different average molar masses and anionicity were synthesized by Reversible Addition Fragmentation chain Transfer / Macromolecular design by interchange of xanthates (RAFT/MADIX) polymerization using a low molar mass hydrophilic macro-RAFT/MADIX agent. ${ }^{41}$ The characteristics of the polymers such as average molar mass, dispersity and the degree of anionicity are gathered in Table 1. The synthesized statistical polymers P(AM-stAA) will be assimilated to hydrolyzed polyacrylamide and noted HPAM-X where X indicates the degree of anionicity. For injectivity experiments, a commercial polymer used in EOR application denoted f-PAM (Flopaam 3630S, $\mathrm{M}_{\mathrm{w}}=2010^{6} \mathrm{~g} / \mathrm{mol}$ with a degree of anionicity of about 25-30 \%) was purchased from SNF. For comparison, a commercial polyacrylamide denoted c-PAM was also purchased from Sigma-Aldrich $\left(\mathrm{M}_{\mathrm{w}}=4.710^{5} \mathrm{~g} / \mathrm{mol}, \mathrm{Đ}=2.6\right)$ 


\section{Table 1}

Characteristics of the acrylamide-based polymers used in this study ${ }^{\mathrm{a}}$

\begin{tabular}{cccc}
\hline Designation & Anionicity (\%) & $\mathbf{M}_{\mathbf{w}}(\mathbf{k g} / \mathbf{m o l})$ & Dispersity Đ \\
\hline PAM & 0 & 167 & 1.11 \\
HPAM-10 & 10 & 181 & 1.09 \\
HPAM-30 & 30 & 149 & 1.07 \\
c-PAM & 0 & 470 & 2.60 \\
f-PAM & $25-30$ & 20000 & -
\end{tabular}

${ }^{\mathrm{a}}$ The three first acrylamide-based polymers were synthesized by RAFT/MADIX polymerization. c-PAM and fPAM refer to commercial polymers from Sigma-Aldrich and SNF, respectively.

\subsection{TOC adsorption measurements}

$4 \mathrm{~g}$ of silica were first mixed in a bottle with $10 \mathrm{~mL}$ of polymer solution with concentrations ranging from $10 \mathrm{ppm}$ to $200 \mathrm{ppm}$. The bottles were left rolling at constant temperature (23 ${ }^{\circ} \mathrm{C}$ ) during 2 hours. The silica was then removed by centrifugation at $6000 \mathrm{~g}$ for $15 \mathrm{~min}$ to extract the interstitial solution. The equilibrium concentration of polymers in the interstitial solution was determined using Total Organic Carbon method (TOC-VCSH, Shimadzu). From this, the amount of polymer adsorbed on mineral phases was computed. The experiments were conducted at the $\mathrm{pH}$ of pure water.

\section{$2.3 N M R$}

${ }^{27} \mathrm{Al}$ magic angle spinning nuclear magnetic resonance (MAS NMR) one-pulse $(\pi / 10)$ spectra were obtained at a frequency of $130.3 \mathrm{MHz}$ on a $4 \mathrm{~mm}$ Bruker probe and an Avance III spectrometer by spinning at $11.5 \mathrm{kHz}$. The pulse duration was $1.5 \mu \mathrm{s}$ and the recycle time was $5 \mathrm{~s}$. Ten thousand scans were accumulated. The chemical shift reference is $\mathrm{Al}^{3+}$ aq in a $0.1 \mathrm{M}$ $\mathrm{AlCl}_{3}$ aqueous solution.

\section{$2.4 X R D$}

X-ray Diffraction (XRD) was performed on a Philips PW 1820 device. Measurement was performed on powdered sample. The diffraction patterns were obtained in the range $2 \theta=5^{\circ}$ to $60^{\circ}$ with a step of $0.02^{\circ}$ and 50 s per step.

\subsection{SEM-EDX}


Scanning electron microscopy and energy dispersive X-ray spectrometer (SEM-EDX) were used to observe the natural silica surface and the synthetic silica beads. Secondary electron images of surface and qualitative X-rays analysis were performed on a FEG Magellan 400 FEI Thermofisher microscope fitted with detector EDX Oxford. The observation takes place at a high acceleration tension of $5 \mathrm{kV}$ and the $\mathrm{X}$-rays analysis at $15 \mathrm{kV}$.

\subsection{Injectivity experiments}

Injectivity was evaluated by measuring the permeability reduction due to polymer adsorption/retention on silica porous discs provided by Heraeus with a nominal maximal pore size ranging between 10 and $16 \mu \mathrm{m}$. Their porosity was measured on a accuPyc II 1340 pycnometer from Micromeritics. The method consists in measuring the pressure loss through the porous disc when injecting water with a constant flow rate before and after injection of a polymer solution. Water or polymer solution were injected at constant flow rate with a syringe pump, model 1000D from Teledyne ISCO. Pressure loss through the porous disc was measured with Rosemount 3051S pressure transducers in the range 0-60 and 0-600 mBar. Two discs were mounted in series in order to prevent retention effects in the second disc. Indeed it is believed that the biggest chains will be trapped in the first porous disc and will no longer affect, or no significantly, the pressure loss measured on the second disc.

The hydrodynamic resistance due to polymer adsorption was measured as follow:

- First, water was injected at a constant flow rate of $15 \mathrm{~mL} / \mathrm{h}$ on the native porous discs, a pressure loss noted $\Delta P_{\mathrm{H}_{2} \mathrm{O}}$, was measured.

- Then, a polymer aqueous solution of f-PAM concentrated at $800 \mathrm{ppm}$ in a brine solution at $6 \mathrm{~g} / \mathrm{L}$ in $\mathrm{NaCl}$, was injected through the media at $15 \mathrm{~mL} / \mathrm{h}$ for $2 \mathrm{~h}$.

- Finally, after $16 \mathrm{~h}$ at rest, water was injected at $15 \mathrm{~mL} / \mathrm{h}$ to flush the polymer solution present in the pores. Pressure was decreasing leading to a pressure drop after stabilization of $\Delta P_{\mathrm{H}_{2} \mathrm{O}}^{\text {after }}$.

Thus, the permeability reduction to water (called residual resistance factor and noted as RRF in the EOR community) due to the irreversible polymer adsorption and retention is defined as $R R F=\frac{\Delta \mathrm{P}_{\mathrm{H}_{2} \mathrm{O}}}{\Delta P_{\mathrm{H}_{2} \mathrm{O}}^{\text {ater }}}$

\section{State of the art: Discrepancy in polyacrylamide adsorption on silica}


Generally, the polymer adsorption depends on three factors: (i) the physicochemical properties of the polymer chains in solution, (ii) the surface state of the absorbent and (iii) the interactions between the polymer chains and the surface. Compared to fundamental studies on the polymer adsorption on solid interface which were mainly performed both in organic solvent and specific conditions, the case of polyacrylamide adsorption on siliceous materials in aqueous solution is more complex. Table 2 lists the various parameters which affect the adsorption of acrylamide-based polymers on siliceous materials. One may observe that, in addition to the intrinsic parameters of both the polymers and the silica surface influencing the adsorption strength, the characteristics of the aqueous solution ( $\mathrm{pH}$, ionic strength) blurs the immediate prognosis on the possibility of adsorption of acrylamide-based polymers on silica.

\section{Table 2}

Parameters influencing the adsorption of acrylamide-based polymers on siliceous materials.

\begin{tabular}{lcc}
\hline & Acrylamide-based polymers & Siliceous surface \\
\hline Average molar mass and dispersity & $\mathrm{X}$ & \\
Anionic groups density & $\mathrm{X}$ & \\
Solution temperature & $\mathrm{X}$ & \\
Solution pH & $\mathrm{X}$ & $\mathrm{X}$ \\
Ionic strength (nature, concentration) & $\mathrm{X}$ & $\mathrm{X}$ \\
Solid/liquid ratio & $\mathrm{X}$ & $\mathrm{X}$ \\
Substrate source & & $\mathrm{X}$ \\
Presence of impurities & & $\mathrm{X}$ \\
Temperature pretreatment & & $\mathrm{X}$ \\
\hline
\end{tabular}

To exemplify the existing lack of clarity concerning the adsorption or not of PAM/HPAM on silica surface, we reported in Table 3 and $\mathbf{4}$ research works showing either an adsorption or a non-adsorption of PAM and HPAM polymers of different average molar masses and dispersity on siliceous materials in various aqueous solutions. Methods used besides TOC, are X-ray photoelectron spectroscopy (XPS), ellipsometry or atomic force microscopy (AFM). In the light of research works where adsorption was observed, there is a common understanding that the PAM/HPAM adsorption on siliceous materials is due to the formation of multiple 
hydrogen bond between the amide groups of the polymers and hydroxyl groups located on the silica surface. ${ }^{29}$ This polymer adsorption process by the formation of multiple hydrogen bonding on silica is similar to other polymer such as poly(vinyl alcohol) $\mathrm{PVA}^{42}$, poly(ethylene glycol) $\mathrm{PEG}^{43}$ and poly(vinylpyrrolidone) $\mathrm{PVP}^{44}$. In order to explain the reported discrepancies, we will discuss the main parameters influencing the adsorption of PAM/HPAM on siliceous materials such as the state of the silica surface state and the physicochemical behavior of the polymer chains in aqueous solution. 


\section{Table 3}

Literature reporting the adsorption of PAM/HPAM on siliceous materials.

\begin{tabular}{|c|c|c|c|c|c|c|}
\hline & Polymer & Substrat & Conditions & Experimental & Results and remarks & References \\
\hline \multirow{10}{*}{$\varepsilon$} & $\begin{array}{c}\text { PAM } \\
M_{\mathrm{w}}=710^{5} \mathrm{~g} / \mathrm{mol} \\
\left(\begin{array}{l}(14 \mathrm{C} \text {-labeled })\end{array}\right.\end{array}$ & $\begin{array}{c}\text { Natural Brazilian quartz } \\
\text { (Prepared by wet grinding and leaching) } \\
0.7 \mathrm{~m}^{2} / \mathrm{g}\end{array}$ & $\begin{array}{l}0.03 \mathrm{M} \mathrm{NaCl} \\
\mathrm{pH}=6-8 \\
\mathrm{~S} / \mathrm{L}=0.03\end{array}$ & $\begin{array}{c}\text { Depletion } \\
\text { Experiment } \\
\left(\mathrm{T}^{\circ}=24 \text { to } 26^{\circ} \mathrm{C}\right)\end{array}$ & $\Gamma_{\mathrm{m}}=0.5-0.6 \mathrm{mg} / \mathrm{m}^{2}$ & $\underset{33}{\text { Lee et al. }}$ \\
\hline & \multirow{5}{*}{$\begin{array}{c}\text { HPAM } \\
\mathrm{M}_{\mathrm{w}}=7.510^{6} \mathrm{~g} / \mathrm{mol} \\
(\mathrm{D}=2.2) \\
\text { Anionicity }=30 \%\end{array}$} & $\begin{array}{c}\text { Silicon carbide }(\mathrm{SiC}) \\
\text { thermal treatment }\end{array}$ & $\begin{array}{c}\mathrm{NaCl}=0.34 \mathrm{M} \\
\mathrm{pH}=5\end{array}$ & \multirow{6}{*}{ TOC } & $\begin{array}{c}\Gamma_{\mathrm{m}} \text { decreasing with temperature of treatment } \\
\quad \Gamma_{\mathrm{m}}=0.4\left(100^{\circ} \mathrm{C}\right) \text { to } \Gamma_{\mathrm{m}}=0 \mathrm{mg} / \mathrm{m}^{2}\left(700^{\circ} \mathrm{C}\right)\end{array}$ & \multirow{6}{*}{$\underset{32}{\text { Lecourtier et al. }}$} \\
\hline & & $\begin{array}{c}\text { Natural sand }\left(99 \% \mathrm{SiO}_{2}\right) \\
0.1 \mathrm{~m}^{2} / \mathrm{g} \\
\end{array}$ & \multirow[b]{2}{*}{$\mathrm{NaCl}=0.34 \mathrm{M}$} & & $\begin{array}{c}\Gamma_{\mathrm{m}} \text { decreasing with pH } \\
\Gamma_{\mathrm{m}}=0.2(\mathrm{pH}=4) \text { to } \Gamma_{\mathrm{m}}=0.05 \mathrm{mg} / \mathrm{m}^{2}(\mathrm{pH}=7.5)\end{array}$ & \\
\hline & & $\begin{array}{c}\text { Silicon Carbide (SiC) } \\
\text { No thermal treatment } \\
1.3 \mathrm{~m}^{2} / \mathrm{g} \\
\end{array}$ & & & $\begin{array}{c}\Gamma_{\mathrm{m}} \text { decreasing with } \mathrm{pH} \\
\Gamma_{\mathrm{m}}=0.7(\mathrm{pH}=4) \text { to } \Gamma_{\mathrm{m}}=0.1 \mathrm{mg} / \mathrm{m}^{2}(\mathrm{pH}=7.5)\end{array}$ & \\
\hline & & $\begin{array}{l}\text { Silicon Carbide (SiC) } \\
\quad 0.1-0.4 \mathrm{~m}^{2} / \mathrm{g}\end{array}$ & $\begin{array}{c}\mathrm{NaCl}=0-4.28 \mathrm{M} \\
\mathrm{pH}=7\end{array}$ & & $\begin{array}{c}\Gamma \mathrm{m} \text { increasing with NaCl} \\
\Gamma_{\mathrm{m}}=0.1(\mathrm{NaCl}=0 \mathrm{M}) \text { to } \Gamma_{\mathrm{m}}=0.5 \mathrm{mg} / \mathrm{m}^{2}(\mathrm{NaCl}=4.28 \mathrm{M})\end{array}$ & \\
\hline & & $\begin{array}{l}\text { Sand } \\
0.1 \mathrm{~m}^{2} / \mathrm{g}\end{array}$ & $\begin{array}{c}\mathrm{NaCl}>0.17 \mathrm{M} \\
\mathrm{pH}=7 \\
\mathrm{NaCl}>0.08 \mathrm{M} \\
\mathrm{pH}=5\end{array}$ & & $\begin{array}{c}\Gamma \mathrm{m} \text { increasing with } \mathrm{NaCl} \\
\Gamma_{\mathrm{m}}=0(\mathrm{NaCl}=0.171 \mathrm{M}) \text { to } \Gamma_{\mathrm{m}}=0.4 \mathrm{mg} / \mathrm{m}^{2}(\mathrm{NaCl}=4.11 \mathrm{M})\end{array}$ & \\
\hline & $\begin{array}{c}\text { PAM } \\
\mathrm{M}_{\mathrm{w}}=7.510^{6} \mathrm{~g} / \mathrm{mol}\end{array}$ & $\begin{array}{c}\text { Sand } \\
\text { Silicon Carbide Non treated }\end{array}$ & $\begin{array}{l}\mathrm{NaCl}=0-4.1 \mathrm{M} \\
\mathrm{pH}=7\end{array}$ & & $\Gamma_{\mathrm{m}}$ constant with $\mathrm{NaCl}$ change & \\
\hline & $\begin{array}{c}\text { HPAM } \\
\mathrm{M}_{\mathrm{w}}=1.510^{3} \mathrm{~g} / \mathrm{mol} \\
\text { Anionicity }=9.3 \% \\
\text { HPAM } \\
\mathrm{M}_{\mathrm{w}}=10^{4} \mathrm{~g} / \mathrm{mol} \\
\text { Anionicity }=14.4 \% \\
\end{array}$ & $\begin{array}{l}\mathrm{SiO}_{2} \text { nanoparticles } \\
326 \mathrm{~m}^{2} / \mathrm{g}\end{array}$ & $\begin{array}{l}\mathrm{NaCl}=0.01 \mathrm{M} \\
\mathrm{pH}=6 \\
\mathrm{~S} / \mathrm{L}=0.003\end{array}$ & Titration & $\begin{array}{l}\Gamma_{\mathrm{m}} \text { decreasing with temperature }\left(15 \text { to } 35^{\circ} \mathrm{C}\right) \\
\Gamma_{\mathrm{m}}=0.4 \mathrm{mg} / \mathrm{m}^{2} \text { to } \Gamma_{\mathrm{m}}=0.2 \mathrm{mg} / \mathrm{m}^{2}\end{array}$ & $\underset{38}{\text { Wisniewska et al. }}$ \\
\hline & $\begin{array}{c}\text { HPAM } \\
\mathrm{M}_{\mathrm{w}}=5.510^{6} \mathrm{~g} / \mathrm{mol} \\
\text { Anionicity }=1 \%\end{array}$ & $\begin{array}{c}\text { Silica nanoparticles (Aerosil; grade } \\
\left.2491 / 380 ; 99.9 \% \mathrm{SiO}_{2}\right) \\
380 \mathrm{~m}^{2} / \mathrm{g} \\
\text { Fresh/Aged/Restored } \\
\end{array}$ & Water & $\begin{array}{l}\text { Titration } \\
\text { And flotation test }\end{array}$ & $\begin{array}{l}\text { Adsorption decreases when silica storage in water duration } \\
\text { increases }\end{array}$ & $\underset{28-30}{\text { Griot et al. }}$ \\
\hline & $\begin{array}{c}\text { PAM } \\
M_{w}=7.510^{6} \mathrm{~g} / \mathrm{mol}\end{array}$ & $\begin{array}{c}\text { Natural sand }\left(99 \% \mathrm{SiO}_{2}\right) \\
0.1 \mathrm{~m}^{2} / \mathrm{g} \\
\end{array}$ & $\begin{array}{c}\mathrm{NaCl}=0-1.7 \mathrm{M} \\
\mathrm{pH}=7\end{array}$ & $\begin{array}{c}\text { TOC } \\
\mathrm{S} / \mathrm{L}=0.02\end{array}$ & PAM adsorption constant with $\mathrm{NaCl}$ concentration & $\underset{35}{\text { Page et al. }}$ \\
\hline
\end{tabular}




\begin{tabular}{|c|c|c|c|c|c|}
\hline $\begin{array}{c}\text { HPAM } \\
M_{w}=7.510^{6} \mathrm{~g} / \mathrm{mol} \\
\text { Anionicity }=30 \%\end{array}$ & & & & $\begin{array}{c}\Gamma_{\mathrm{m}}=0.17 \mathrm{mg} / \mathrm{m}^{2}\left(90^{\circ} \mathrm{C}\right) ; \Gamma_{\mathrm{m}}=0.38 \mathrm{mg} / \mathrm{m}^{2}\left(30^{\circ} \mathrm{C}\right) \\
\text { HPAM adsorption increases with salt concentration } \\
\mathrm{T}=30^{\circ} \mathrm{C}: \Gamma_{\mathrm{m}}=0(\mathrm{NaCl}=0.085 \mathrm{M}) \text { to } \Gamma_{\mathrm{m}}=0.25 \mathrm{mg} / \mathrm{m}^{2}(\mathrm{NaCl}=1.7 \mathrm{M}) \\
\mathrm{T}=90^{\circ} \mathrm{C}: \Gamma_{\mathrm{m}}=0(\mathrm{NaCl}=0.085 \mathrm{M}) \text { to } \Gamma_{\mathrm{m}}=0.08 \mathrm{mg} / \mathrm{m}^{2}(\mathrm{NaCl}=1.7 \mathrm{M})\end{array}$ & \\
\hline $\begin{array}{c}\text { HPAM } \\
M_{\mathrm{w}}=810^{6} \mathrm{~g} / \mathrm{mol} \\
\text { Anionicity }=10 \%\end{array}$ & Quartz and glass slides & $\mathrm{pH}=2-10$ & $\begin{array}{l}\text { X-ray photoelectron } \\
\text { spectroscopy }\end{array}$ & Adsorption independent of $\mathrm{pH}$ & $\underset{27}{\text { Graveling et al. }}$ \\
\hline $\begin{array}{c}\text { PAM } \\
\mathrm{M}_{\mathrm{w}}>210^{6} \mathrm{~g} / \mathrm{mol}\end{array}$ & $\begin{array}{c}\text { Silicon wafer } \\
\text { (Thermally oxidized) }\end{array}$ & $\begin{array}{c}\mathrm{KCl}=0.1 \mathrm{M} \\
\mathrm{pH}=7\end{array}$ & Ellipsometry & $\Gamma_{\mathrm{m}}=0.15 \mathrm{mg} / \mathrm{m}^{2}$ & Samoshina et al. ${ }^{3}$ \\
\hline $\begin{array}{c}\text { PAM } \\
\mathrm{M}_{\mathrm{w}}=7.510^{6} \mathrm{~g} / \mathrm{mol} \\
\text { HPAM } \\
\mathrm{M}_{\mathrm{w}}=7.510^{6} \mathrm{~g} / \mathrm{mol} \\
\text { Anionicity }=27 \%\end{array}$ & $\begin{array}{l}\text { Sand }\left(99 \% \mathrm{SiO}_{2}\right) \\
\quad 0.03 \mathrm{~m}^{2} / \mathrm{g}\end{array}$ & $\begin{array}{c}9<\mathrm{pH}<12.5 \\
\mathrm{NaCl}=0.17 \mathrm{M} \\
\mathrm{T}=30^{\circ} \mathrm{C}\end{array}$ & $\begin{array}{c}\text { TOC } \\
\mathrm{S} / \mathrm{L}=0.01\end{array}$ & $\begin{array}{c}\text { PAM, } \Gamma \mathrm{m} \text { decreasing with the } \mathrm{pH} \\
\Gamma_{\mathrm{m}}=0.35(\mathrm{pH}=9) \text { to } \Gamma_{\mathrm{m}}=0 \mathrm{mg} / \mathrm{m}^{2}(\mathrm{pH}=12.5) \\
\text { HPAM, No adsorption }\end{array}$ & $\underset{31}{\text { Guevellou et al. }}$ \\
\hline $\begin{array}{c}\text { PAM } \\
\mathrm{M}_{\mathrm{w}}=1810^{6} \mathrm{~g} / \mathrm{mol}\end{array}$ & $\begin{array}{c}\text { Glass bead } \\
(25 \mu \mathrm{m})\end{array}$ & $\begin{array}{c}\mathrm{NaCl} \\
(0.01 \text { and } 0.34 \mathrm{M})\end{array}$ & AFM & Adsorption kinetics increasing with salt & Al-Hashmi et al. \\
\hline $\begin{array}{c}\text { PAM } \\
M_{w}=610^{6} \mathrm{~g} / \mathrm{mol}\end{array}$ & $\begin{array}{c}\text { Glass bead } \\
(25 \mu \mathrm{m})\end{array}$ & $\begin{array}{c}\mathrm{KCl}, \mathrm{LiCl} \mathrm{KNO}_{3} \\
0.27 \mathrm{M} \\
\mathrm{pH}=6.5 \\
\mathrm{~T}=22^{\circ} \mathrm{C}\end{array}$ & TOC and AFM & $\begin{array}{l}\text { Weak adsorption }\left(\Gamma_{\mathrm{m}}=0.01 \mathrm{mg} / \mathrm{m}^{2}\right) \\
\text { Adsorption kinetics dependence with cation nature }\end{array}$ & $\underset{23}{A l-H a s h m i ~ e t ~ a l . ~}$ \\
\hline $\begin{array}{c}\text { HPAM } \\
\mathrm{M}_{\mathrm{w}}=10-1510^{6} \\
\mathrm{~g} / \mathrm{mol} \\
\text { Anionicity }=21 \%\end{array}$ & $\begin{array}{l}\text { Fine silica sand } \\
\text { (size }<0.15 \mathrm{~mm} \text { ) }\end{array}$ & $\begin{array}{c}\mathrm{NaCl} \\
(0-0.01 \mathrm{M}) \\
\mathrm{CaCl}_{2} \\
(0-0.005 \mathrm{M})\end{array}$ & $\begin{array}{c}\text { Titration } \\
\mathrm{S} / \mathrm{L}=0.005-0.02\end{array}$ & $\begin{array}{l}\text { Adsorption increases with salt concentration } \\
\mathrm{Ca}^{2+} \text { more effective than } \mathrm{Na}^{+}\end{array}$ & $\underset{34}{\text { Lu et al. }}$ \\
\hline $\begin{array}{c}\text { PAM } \\
\mathrm{M}_{\mathrm{w}}=1810^{6} \mathrm{~g} / \mathrm{mol} \\
\text { HPAM } \\
\mathrm{M}_{\mathrm{w}}=1810^{6} \mathrm{~g} / \mathrm{mol} \\
\text { Anionicity }=27.8 \%\end{array}$ & Silica crystals (QCM-D) and glass beads & $\begin{array}{l}\mathrm{KCl}(0.27 \mathrm{M}) \\
\mathrm{pH} 6.5\end{array}$ & $\begin{array}{l}\text { AFM } \\
\text { quartz crystal } \\
\text { microbalance }\end{array}$ & $\begin{array}{l}\text { Slow adsorption kinetics } \\
\text { PAM: } \Gamma_{\mathrm{m}}=2.7 \mathrm{mg} / \mathrm{m}^{2} \text { after } 7 \mathrm{~h} \\
\text { HPAM: } \Gamma_{\mathrm{m}}=2.3 \mathrm{mg} / \mathrm{m}^{2} \text { after } 7 \mathrm{~h}\end{array}$ & Al-Hashmi et al. \\
\hline $\begin{array}{c}\text { PAM } \\
\mathrm{M}_{\mathrm{w}}=0.15-1510^{6} \\
\mathrm{~g} / \mathrm{mol} \\
\mathrm{HPAM} \\
\mathrm{M}_{\mathrm{w}}=2010^{6} \mathrm{~g} / \mathrm{mol} \\
\text { Anionicity }=30 \% \\
\end{array}$ & Glass capillary & $\begin{array}{l}\text { Brine solution } \\
\quad(5-10 \mathrm{wt} \%) \\
\mathrm{NaCl} / \mathrm{CaCl}_{2}(9 / 1)\end{array}$ & AFM & Measurements of the adsorbed polymer layer & Grattoni et al. ${ }^{26}$ \\
\hline $\begin{array}{c}\text { HPAM } \\
M_{w}=6-810^{6} \mathrm{~g} / \mathrm{mol} \\
\text { Anionicity }=30 \%\end{array}$ & $\begin{array}{l}\text { Quartz particles } \\
(420-590 \mu \mathrm{m})\end{array}$ & $\begin{array}{c}\text { Brine solution } \\
\left(0.22 \% \mathrm{CaCl}_{2}, 2.3 \%\right. \\
\mathrm{NaCl})\end{array}$ & TOC & $\Gamma_{\mathrm{m}}=0.12-0.15 \mathrm{mg} / \mathrm{m}^{2}$ & Wan et al. ${ }^{45}$ \\
\hline
\end{tabular}




\begin{tabular}{|c|c|c|c|c|c|}
\hline $\begin{array}{c}\text { PAM } \\
\mathrm{M}_{\mathrm{w}}=16710^{3} \mathrm{~g} / \mathrm{mol} \\
(Đ=1.11) \\
\text { HPAM } \\
\mathrm{M}_{\mathrm{w}}=18110^{3} \mathrm{~g} / \mathrm{mol} \\
(Đ=1.09) \\
\text { Anionicity }=10 \% \\
\text { HPAM } \\
\mathrm{M}_{\mathrm{w}}=14910^{3} \mathrm{~g} / \mathrm{mol} \\
(Đ=1.07) \\
\text { Anionicity } 30 \% \\
\mathrm{HPAM} \\
\mathrm{M}^{\mathrm{w}}=1810^{6} \mathrm{~g} / \mathrm{mol} \\
\text { Anionicity=30\% } \\
\text { (see table } 1 \text { ) }\end{array}$ & $\begin{array}{c}\text { Crystallized natural quartz (Sikaisol) } \\
0.56 \mathrm{~m}^{2} / \mathrm{g} \\
\text { Glass porous filter } \\
\text { (see experimental part) }\end{array}$ & $\begin{array}{l}\mathrm{CaCl}_{2} \\
\mathrm{NaCl}\end{array}$ & $\begin{array}{c}\text { TOC } \\
\left(T=22^{\circ} \mathrm{C}\right) \\
\mathrm{S} / \mathrm{L}=0.4\end{array}$ & $\begin{array}{l}\text { PAM: Adsorption on Sikaisol }\left(\Gamma_{\mathrm{m}}=0.4 \mathrm{~g} / \mathrm{m}^{2}\right) \\
\text { Adsorption decreases with anionicity } \\
\text { HPAM: Adsorption increases with ionic strength }\end{array}$ & This Work \\
\hline
\end{tabular}

\section{Table 4}

Literature reporting no adsorption of PAM/HPAM on siliceous materials.

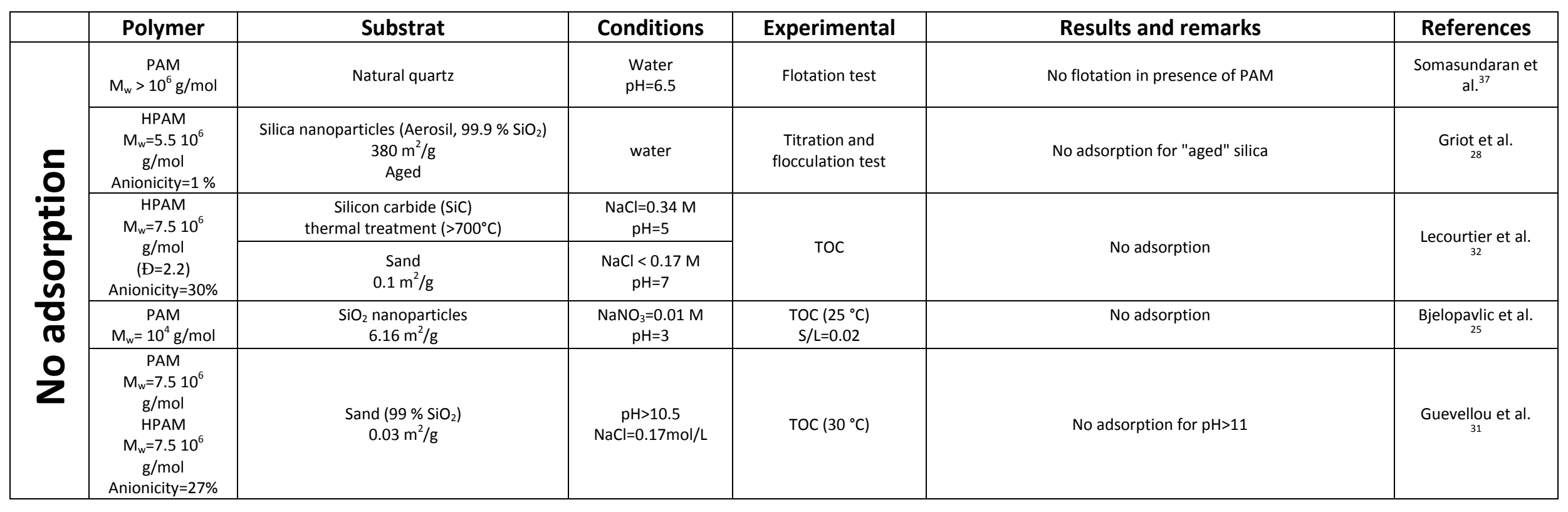




\begin{tabular}{|c|c|c|c|c|c|}
\hline $\begin{array}{c}\text { PAM } \\
\mathrm{M}_{\mathrm{w}}=4.710^{5} \\
\mathrm{~g} / \mathrm{mol} \\
(\mathrm{D}=2.6)\end{array}$ & $\begin{array}{c}\text { Synthetic silica particles } \\
1.1 \mathrm{~m}^{2} / \mathrm{g} \\
\text { (see experimental part) }\end{array}$ & $\begin{array}{l}\text { Water } \\
\mathrm{pH}=2\end{array}$ & $\begin{array}{c}\text { TOC } \\
\left(\mathrm{T}=22^{\circ} \mathrm{C}\right) \\
\mathrm{S} / \mathrm{L}=0.4\end{array}$ & No adsorption & This work \\
\hline
\end{tabular}




\subsection{Chemical surface state of siliceous materials}

As the adsorption of PAM/HPAM on silica surface is induced by the formation of hydrogen bonds, the density and the accessibility of the silanol groups is crucial. Consequently, a large amount of literature has been devoted to study the surface of silica by $\mathrm{NMR}^{46-49}$ and $\mathrm{IR}^{50-55}$. Silanol groups can be classified according to their coordination (single or geminal) and their ability to interact or not with neighboring silanols (isolated or vicinal). ${ }^{56}$ In addition to silanols groups, siloxane groups are also present on the silica surface. According to the origin of silica, purity, surface treatment, storage condition, the density and accessibility of both silanol and siloxane groups strongly differ from one surface to another. Then, for siliceous materials the changes in surface groups composition obviously affect its reactivity and consequently its affinity with polymers. The silanol density is mainly dependent on the silica type. Tuel et al. studied the surface properties of pyrogenic and precipitated silica by solid state $\mathrm{NMR}^{57}$. They showed that pyrogenic silica has a low silanol density at the surface ( 2 to $4 \mathrm{SiOH} / \mathrm{nm}^{2}$ ) while precipitated silica has a higher silanol density $\left(7\right.$ to $\left.8 \mathrm{SiOH} / \mathrm{nm}^{2}\right)$ in agreement with the average value of $5 \mathrm{SiOH} / \mathrm{nm}^{2}$ reported by Zhuravlev. ${ }^{58}$ Then, the surface composition of silica can also be modified by thermal treatment. ${ }^{59,60}$ According to literature, the behavior of the surface silica hydroxyls as a function of thermal treatment temperature can be summarized in Figure 1. ${ }^{60}$ An increase of temperature results in a decrease of total silanol density at the surface of silica. The neighboring silanol groups condense and form a siloxane bridge inducing a more hydrophobic character to the siliceous material. However, the density of free silanol groups first increases as dehydroxylation of hydrogen bonded silanol groups creates supplementary isolated silanols. The reverse rehydroxylation process is not always possible. According to reported literature ${ }^{53,61,62}$, the reversibility of this phenomenon depends on the temperature of the thermal treatment. While complete rehydroxylation is possible after dehydroxylation at moderate temperatures, a part of the dehydroxylation was irreversible at the highest temperatures. Lecourtier et al. investigated the adsorption behavior of a hydrolyzed and of a neutral polyacrylamide on siliceous material treated at different temperatures. $^{32}$ Figure 1c shows a drastic decrease in polymer adsorption when the temperature of thermal treatment increases. An absence of HPAM adsorption is observed onto the siliceous surface treated at $700{ }^{\circ} \mathrm{C}$. The depletion of silanol groups as observed in Figure 1 could explain the radical changes of polyacrylamide affinity toward the same siliceous materials. The correlation between the depletion of silanol and decrease in polyacrylamide adsorption suggest that silanol groups are the anchoring sites. 

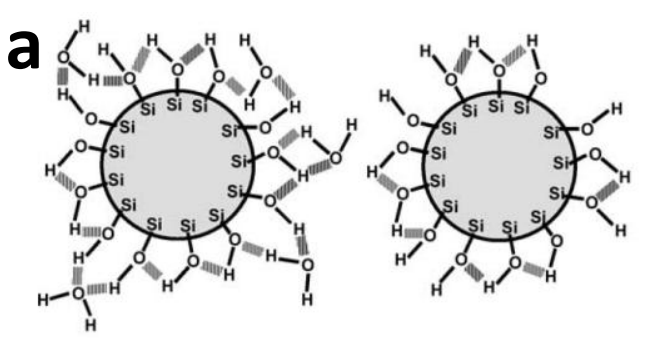

$\underline{190}{ }^{\circ} \mathrm{C}$

Hydrated silica under atmospheric storage conditions

Fully hydroxylated silica $\Gamma_{\text {free Si-OH }}=1.8 \mu \mathrm{mol} / \mathrm{m}^{2}$

$\Gamma_{\text {H-bound Si-OH }}=5.8 \mu \mathrm{mol} / \mathrm{m}^{2}$

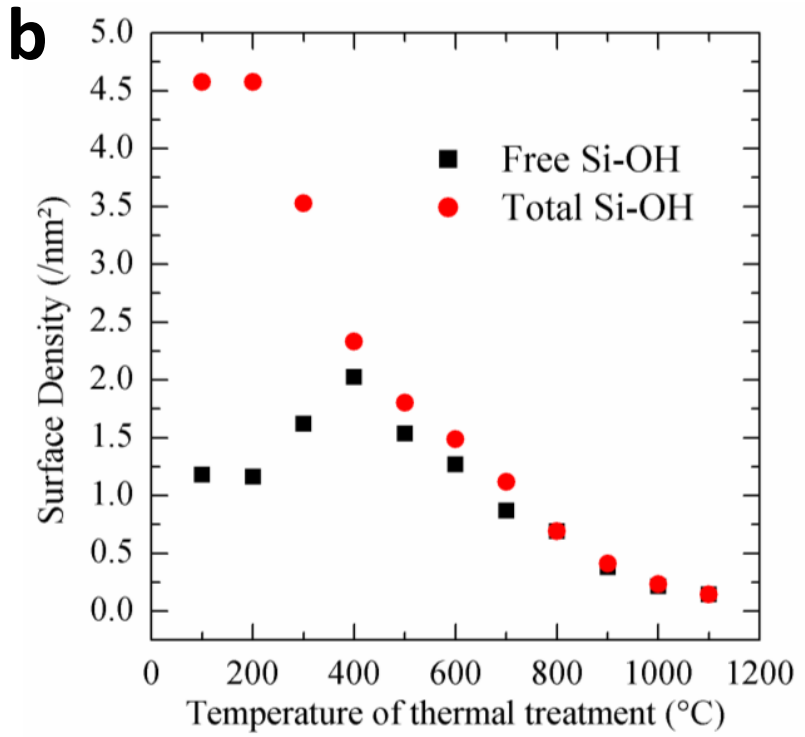

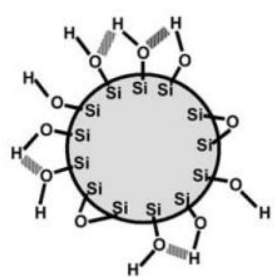
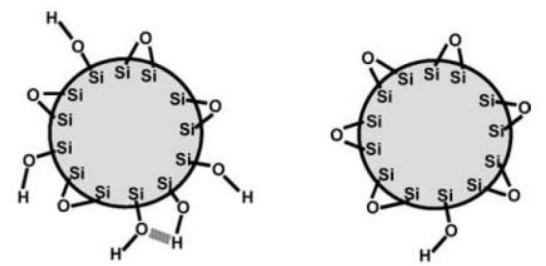

$\underline{400}{ }^{\circ} \mathrm{C}$

$\underline{700}{ }^{\circ} \mathrm{C}$

$\geq 1000^{\circ} \mathrm{C}$
H-bound $\mathrm{Si}-\mathrm{OH}$ ע H-bound $\mathrm{Si}-\mathrm{OH}$

free $\mathrm{Si}-\mathrm{OH} \pi$ free $\mathrm{Si}-\mathrm{OH} \boldsymbol{У}$

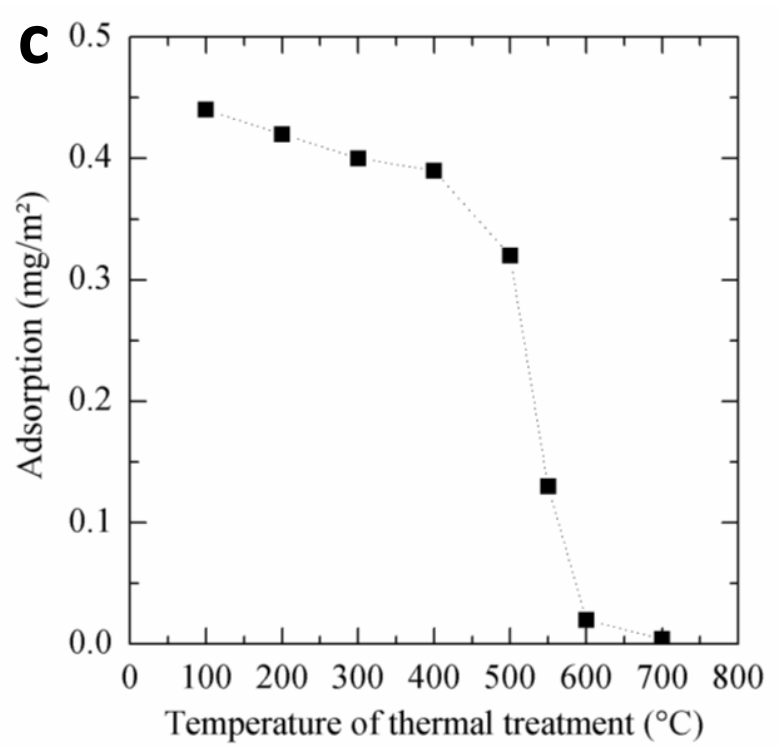

Fig. 1. Impact of chemical state of siliceous material. (a) Schematic representation of the distinct silanol groups onto the silica surface as a function of the thermal treatment. Reprinted from Dugas et al. ${ }^{60}$ (b) Variation of the free silanol groups density compared to the total amount of silanol groups as a function of the thermal treatment. Adapted from Dugas et al. ${ }^{60}$ (c) Impact of the thermal treatment of the adsorption of HPAM $\left(\mathrm{M}_{\mathrm{w}}=7.610^{6} \mathrm{~g} / \mathrm{mol}, 30 \%\right.$ of anionicity) on silicon carbide with an oxidized surface. Adapted from Lecourtier et al. ${ }^{32}$

\subsection{Storage conditions of siliceous materials}

Storage and conditioning of siliceous materials can also change the surface reactivity of these materials. Several studies have shown a decrease in the affinity of low hydrolyzed PAM to the surface of siliceous materials after immersion in aqueous solution. ${ }^{28-30}$ Indeed, as a function of 
immersion time, the authors observed a considerable decrease of PAM adsorption on thermal silica (Aerosil). ${ }^{28}$ The Figure 2 shows the adsorption isotherm of PAM onto "fresh", "aged" and "restored" thermal silica. "Fresh" silica corresponds to an anhydrous silica freshly mixed with water whereas "aged" silica was stored in aqueous solution until it becomes completely non-flocculable by polyacrylamide (i.e. no polyacrylamide adsorption). The time required for silica to become "aged" in water is of few hours and it increases when $\mathrm{pH}$ decreases. ${ }^{29}$ Finally, "restored" silica corresponds to an aged silica heated for 1 to 4 days at $300{ }^{\circ} \mathrm{C}$ or above. The process of ageing in the presence of water could be at the origin of the removal of anchoring surface sites on which polyacrylamide adsorbs. Heating the silica suspension at 300 ${ }^{\circ} \mathrm{C}$ or above re-generates these active sites. Infra-red (IR) spectra of aged silica show a disappearance of the peak ascribed to free silanol groups while it appears again when the silica is restored. According to Griot et al., these changes on free silanol group density at the silica surface could explain the drastic changes in polymer adsorption. ${ }^{28}$ Here again the correlation between the presence of free silanol groups onto the silica surface and the adsorption of polyacrylamide suggests that silanol groups are the anchoring site. However the mechanism at the origin of silica surface modification when stored in aqueous medium has not yet been understood.

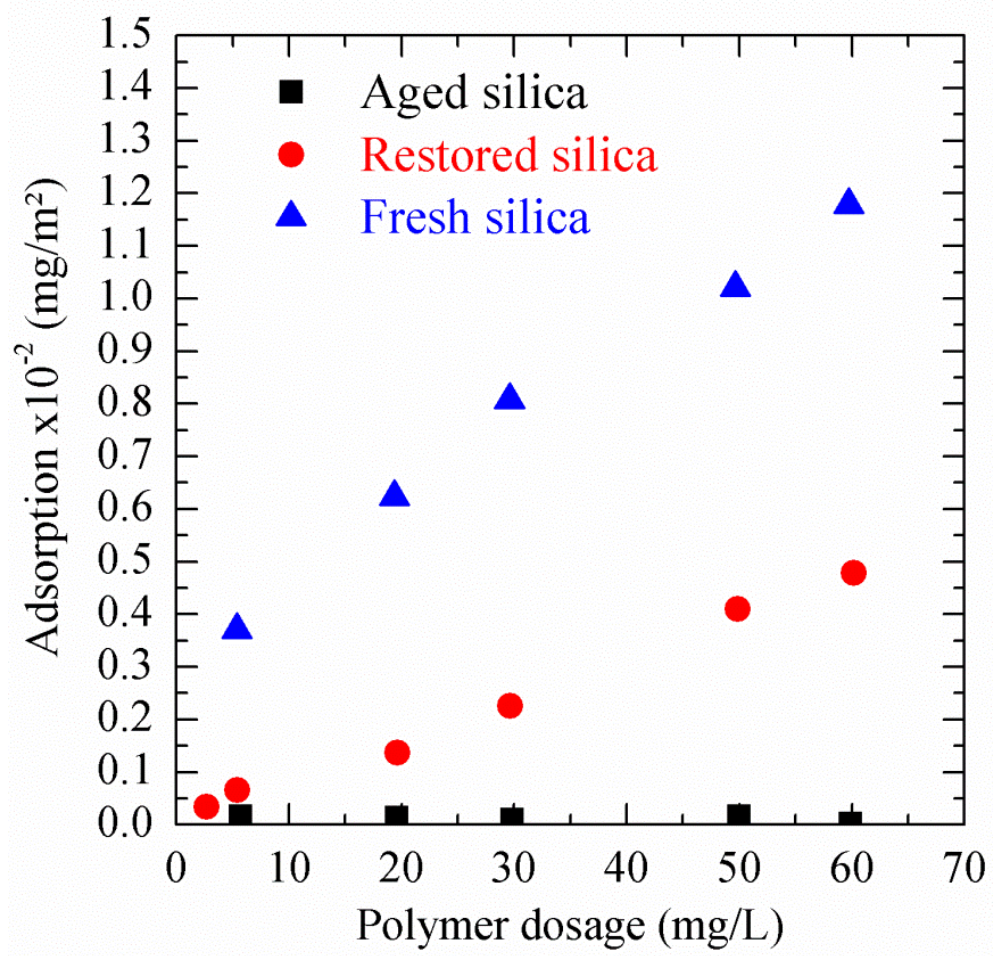

Fig. 2. Impact of the storage condition on polyacrylamide adsorption. Adsorption isotherm of 
PAM $\left(\mathrm{M}_{\mathrm{w}}=5.510^{6} \mathrm{~g} / \mathrm{mol}, 1 \%\right.$ of anionicity) on "fresh", "aged" and "restored" silica particles. Adapted from Griot et al. ${ }^{28}$

\subsection{Surface purity of siliceous materials}

As a function of their origin (natural or synthetic), the surface of siliceous materials can support in some cases a few amount of other oxides. According to their density, these oxides can strongly condition the macroscopic properties of siliceous materials (charge, solvation, acidity, hydrophobicity) and offer possible new sites for adsorption process. Nevertheless, a thorough chemical analysis of the purity of the surface of siliceous materials is rarely performed in the literature before conducting polymer adsorption measurements.

Pefferkorn et al. were the first to show the occurrence of a strong adsorption of neutral PAM on natural (kaolinite) and synthetic aluminosilicates. ${ }^{63}$ They observed that PAM does not adsorb on the basal face of kaolinite but only on the edge face where aluminol sites are located. Afterwards, they deeply investigated the effect of the aluminum grafting onto the silica surface on the polyacrylamide adsorption. ${ }^{64,65}$ They clearly established that the presence of minute amounts of aluminol sites on the surface of siliceous materials can considerably affect the amount of adsorbed polyacrylamide. Figure 3 summarizes the results obtained by Pefferkorn et al. showing that at a fixed polymer concentration in solution, the adsorbed amount increases linearly with the degree of aluminum grafting. Note that in this case, no polyacrylamide adsorption has been measured on the pure silica. The authors thus established that hydrogen bonds between amide groups and aluminol ones dominate polyacrylamide adsorption compared to adsorption on silanol groups. This important conclusion was supported by a decrease of the adsorbed amount when the solution $\mathrm{pH}$ was increasing thus breaking the hydrogen bonds responsible for adsorption. 


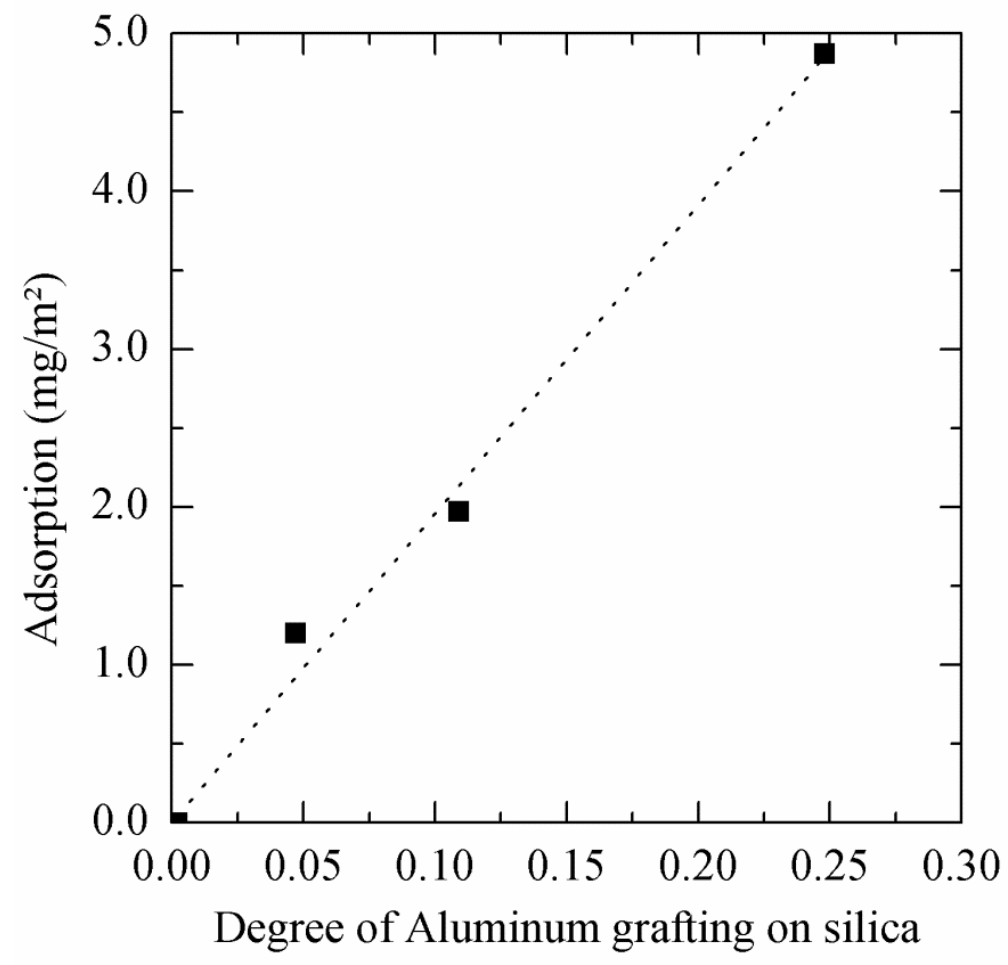

Fig. 3. Adsorption of polyacrylamide on siliceous material as a function of the grafting degree of siliceous material by aluminum. Adapted from Pefferkorn et al. ${ }^{64,65}$

The increase of adsorption ability as a function of oxide impurity was confirmed by Gravelling et al. who compared the adsorption of HPAM on quartz, feldspar and kaolinite having respectively an increasing aluminum content. ${ }^{27}$ They showed that kaolinite adsorbs 3 times more of HPAM than quartz. On the other hand, for their part the authors observed the HPAM adsorption on quartz taken as pure silica material without verifications. It remains that the presence of impurities such as aluminol groups on the surface of what is nominally reported as quartz or silica is a determining factor for polyacrylamide that is too often overlooked. 


\subsection{Impact of physicochemical parameters ( $\mathrm{HH}$, ionic strength and temperature) on the polymer adsorption process}

The $\mathrm{pH}$ of the solution is a crucial parameter in order to investigate the adsorption of acrylamide-based polymers on siliceous materials. Indeed, the $\mathrm{pH}$ changes both the chemical surface state of the siliceous materials and the charge of the polymers particularly in the case of HPAM and can consequently influence the adsorption process. In the case of silica and generally for oxides, the amount of neutral $\mathrm{MOH}$ groups onto the surface depends on the point of zero charge (PZC). It is a consequence of the ionization reactions of the surface hydroxyl groups represented by:

$$
\begin{gathered}
\mathrm{MOH}+\mathrm{H}^{+} \rightarrow \mathrm{MOH}_{2}^{+} \\
\mathrm{MOH}+\mathrm{HO}^{-} \rightarrow \mathrm{MO}^{-}+\mathrm{H}^{+}
\end{gathered}
$$

The driving force of the adsorption of acrylamide-based polymers on siliceous materials would be the formation of multiple hydrogen bonds between the amide groups of the polymers and neutral MOH groups on the surface. ${ }^{29}$ Hence the adsorbed polymer amount is expected to be higher when the $\mathrm{pH}$ is close or lower to the oxide $\mathrm{PZC}$, i.e. when the density of $\mathrm{MOH}$ is the highest. Figure 4a reports on the investigation of the PAM adsorption on several oxides with different PZC at $\mathrm{pH}$ from 6 to $8 .^{33}$ The authors observed convincingly that the higher the PZC of the mineral oxide is, the higher is the adsorbed PAM amount. They also noticed that PAM adsorption is considerably affected when the $\mathrm{pH}$ is higher than the PZC. Indeed, when the $\mathrm{pH}$ of the solution is higher than the PZC, the density of anchoring surface groups, $\mathrm{MOH}$, decreases. This observation was supported by Guevellou et al. who clearly shown a decrease of PAM adsorption on silica as a function of $\mathrm{pH}$ from $\Gamma_{\mathrm{m}}=0.35$ at $\mathrm{pH}=9$ to $\Gamma_{\mathrm{m}}=0 \mathrm{mg} / \mathrm{m}^{2}$ at $\mathrm{pH}=12.5$ (Figure 4b). ${ }^{31}$ The authors indicated that despite a $\mathrm{pH}$ value of 9 , some silanol groups must persist. It should be mentioned here that at high $\mathrm{pH}$, around 10 , the polymer can undergo hydrolysis and introduce in the system electrostatic forces which could be at the origin of the drastic decrease in PAM adsorption. 

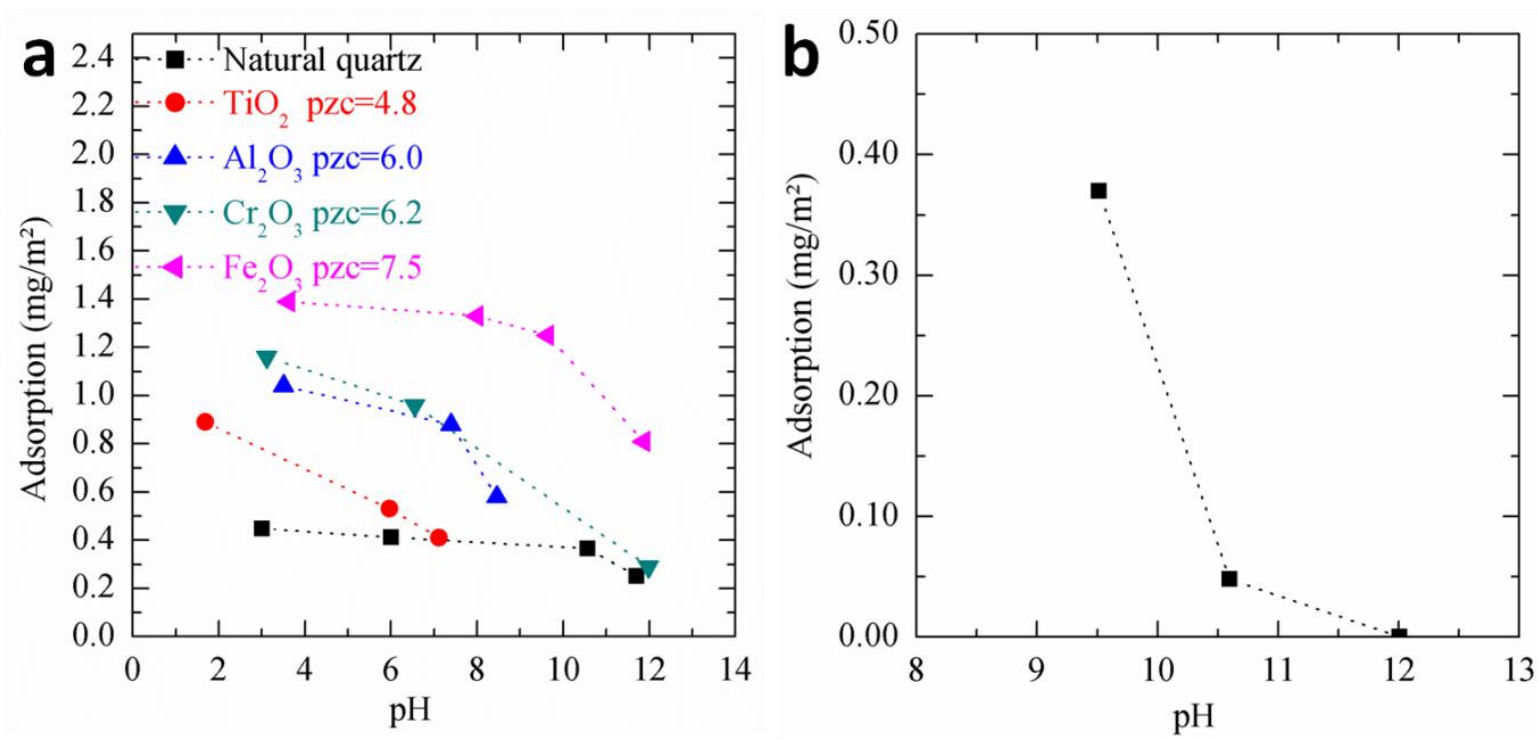

Fig. 4. Impact of the $\mathrm{pH}$ on the adsorption process. (a) Adsorption isotherm of neutral polyacrylamide, on several oxides of different PZC (adapted from Lee et al.) ${ }^{33}$ and adsorption of c-PAM on natural quartz (present work), (b) Variation of the absorbed PAM amount on silica material as a function of the $\mathrm{pH}$. Adapted from Guevellou et al. ${ }^{31}$

After $\mathrm{pH}$, the ionic strength has also a significant influence on the behavior of the polymer chains in solution notably for partially hydrolyzed polyacrylamide HPAM. Indeed HPAM chains bear carboxylic groups which turn to negatively charged carboxylate groups when increasing the $\mathrm{pH}$. The repulsive interactions between the negative charge of the polymer chains and the negative charge onto the siliceous material surface can prevent the adsorption of HPAM. However, an increase of the ionic strength screens the electrostatic repulsive forces and promotes the adsorption of HPAM on siliceous materials. ${ }^{32,34,35}$ On the other hand, the temperature can also modulate the strength of the interactions between adsorbed PAM/HPAM and siliceous materials. Indeed, several authors reported a decrease of the adsorbed PAM or HPAM amount on synthetic or natural siliceous materials when increasing the temperature. ${ }^{35}$, ${ }^{38}$ Consequently, the reported results suggest that the adsorption of HPAM on siliceous materials is not only promoted by attractive hydrogen bonding forces between the amide group and the neutral hydroxyl groups at the surface of the mineral but also moderated by the repulsion between the carboxylate groups and the negative groups at the surface.

In summary, we can conclude from the literature that (i) acrylamide-based polymers adsorb on silica surface by the formation of multiple hydrogen bonding between the polymer and the silica surface groups. (ii) The adsorption on pure silica is weak, strongly dependent on both 
the physicochemical parameters of the silica/polymer system (Table 2) and the resolution of the experimental techniques used for detection. (iii) The adsorption is heightened by the presence of impurities which is not taken into account or ignored in most of the research studies.

In addition to the literature cited above (Table 3), we can also cited works which clearly show the interaction of silica nanoparticles with acrylamide-based polymers without quantification using electroacoustic spectroscopy or rheology confirming the adsorption of PAM/HPAM on silica surface. ${ }^{66-69}$

\section{Present results}

While the literature clearly identifies adsorption as a determining factor for polymer flows in porous media $^{15,26,70,71}$, the diversity of conditions susceptible to impact critically adsorption blurs the issue. Again, this is exemplified in Table $\mathbf{3}$ and $\mathbf{4}$ which summarize the literature and show conflicting reports on the adsorption or not of PAM/HPAM on siliceous materials. In order to clarify the relation between adsorption and flow reduction, we thus first performed a set of experiments on the adsorption of PAM/HPAM on various siliceous materials. For acrylamide-based polymers, we used commercial PAM/HPAM but also PAM/HPAM synthesized by RAFT/MADIX polymerization (see Table 1). The synthesized polymers allowed us to well control the degree of anionicity from 0 to $30 \%$ while keeping a similar average molar mass and a low dispersity. For siliceous materials, crystallized natural quartz, synthetic amorphous silica particles and siliceous porous discs were employed (see experimental part). The latter sample was chosen to perform the injectivity experiments. Results of static adsorption tests are presented in Figure 5. 

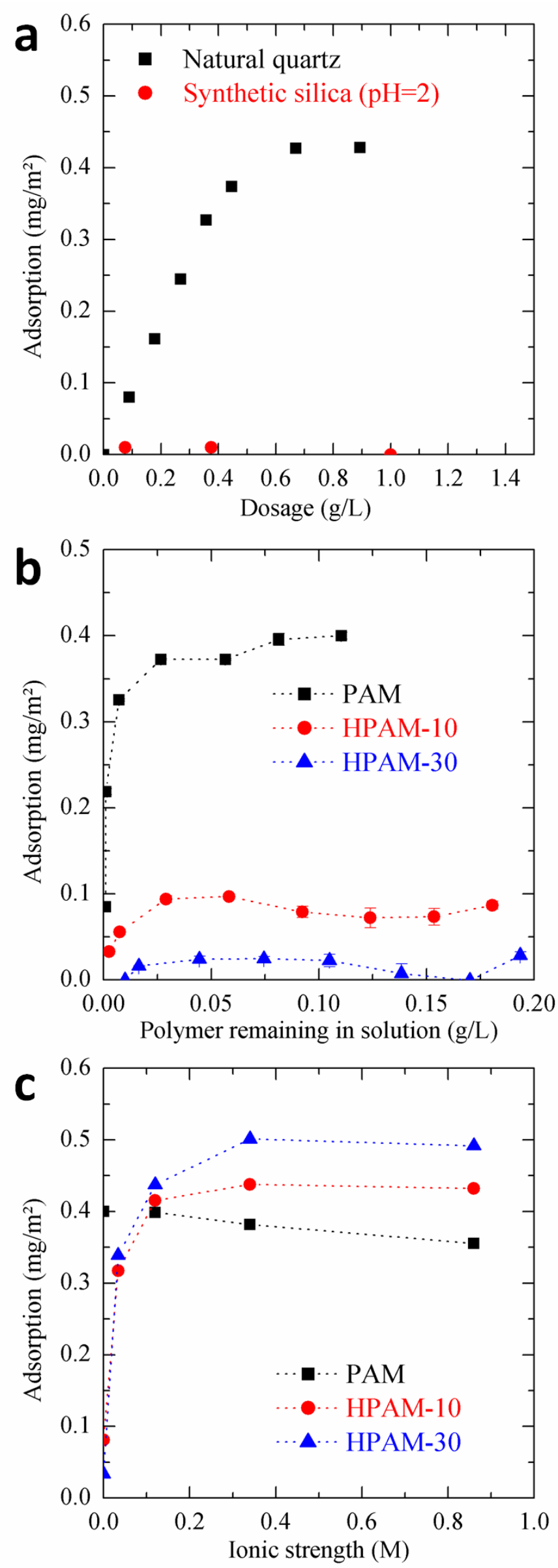
Fig. 5. Adsorption of PAM/HPAM on siliceous material. (a) Adsorption isotherm performed at $\mathrm{pH} 2$ of c-PAM on natural quartz and synthetic silica (b) Adsorption isotherms of acrylamide-based polymers as a function of the anionicity degree (PAM, HPAM-10 and HPAM-30) on natural quartz (c) Adsorption isotherms of acrylamide-based polymers with different anionicity degree (PAM, HPAM-10 and HPAM-30) on natural quartz as a function of the ionic strength $\left(\mathrm{CaCl}_{2}\right)$ at $\mathrm{pH}=8.7$.

Firstly, the adsorption isotherms of neutral PAM (c-PAM) on natural and synthetic silica materials were determined in water at pH 2 and given in Figure 5a. We observed that neutral c-PAM adsorbs on natural quartz with an adsorbed amount at the plateau of $0.45 \mathrm{mg} / \mathrm{m}^{2}$ while no adsorption was detectable on synthetic silica particles. In Figure 5b, c, all the parameters influencing the adsorption of PAM/HPAM such as the thermal treatment, the anionicity degree of polymers, the ionic strength and the $\mathrm{pH}$ on siliceous materials (synthetic silica and natural quartz) were explored except the temperature. As reported in different research works mentioned in the Table 3, we observed that (i) neutral PAM adsorption is independent of the ionic strength (Figure 5c) (ii) concerning HPAM, the polymer adsorption depends on the anionicity degree of the polymers (Figure $\mathbf{5 b}, \mathbf{c}$ ). ${ }^{31}$ Indeed, the adsorption of HPAM is lower than the one for neutral PAM at low ionic strength. However, the polymer adsorption was restored by increasing the salt concentration. The adsorbed amount at the isotherm plateau was then similar to the one obtained for neutral PAM (Figure 5c). ${ }^{32}$ These experiments efficiently supported the conclusion that the driving force of the acrylamide-based polymers was induced by multiple hydrogen bonding between the polymer and the silica surface. However, at this stage of the analysis we do not explain why PAM or HPAM do not adsorb on synthetic silica particles while they strongly adsorb on the natural quartz sample. In order to investigate the difference between these two siliceous materials, we thoroughly characterized the two materials using XRD, SEM coupled with EDX analysis and ${ }^{27} \mathrm{Al}$ NMR. The characterization results are given in Figure 6. As shown in Figure 6a, only quartz was identified in the X-ray diffraction of Sikaisol, the natural quartz sample. However, its analysis by ${ }^{27} \mathrm{Al}$ NMR showed the presence of a high amount of octahedral aluminum thus suggesting the presence of surface impurities in the form of aluminol at the surface of the natural quartz sample (Figure 6b). The presence of aluminum onto the quartz surface was confirmed by EDX analysis on SEM images (Figure 6e and $\mathbf{f}$ ). 

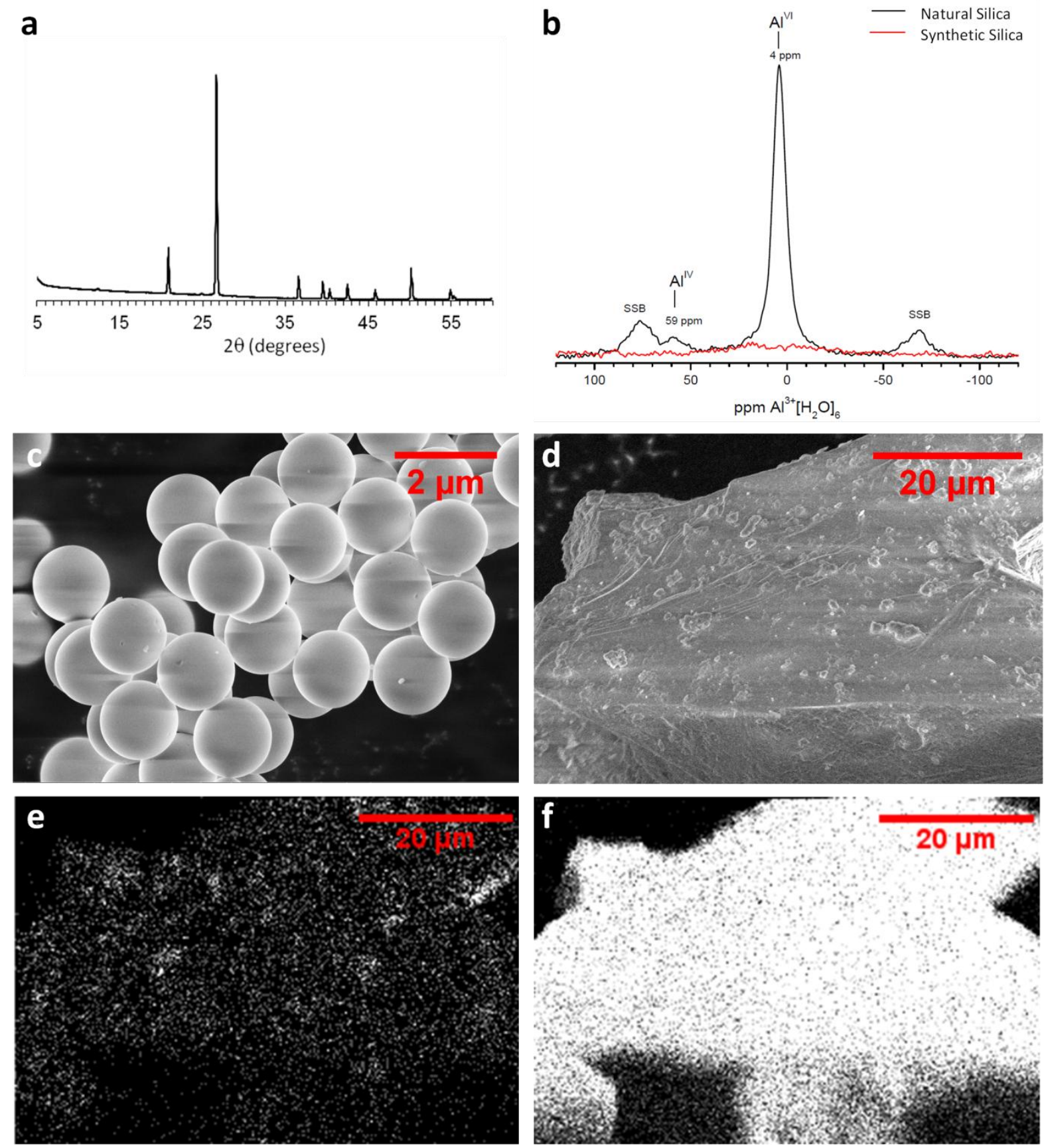

Fig. 6. Characterization of the amorphous synthetic and crystallized natural silica materials. (a) X-ray diffraction of natural quartz. Quartz was identified as the main crystalline component. (b) ${ }^{27} \mathrm{Al}$ NMR of both natural (Sikaisol) and synthetic silica materials. (c, d) SEM images of both natural (Sikaisol) and synthetic silica materials. (e, f) Aluminum and silicium analysis of natural quartz by EDX analysis. 
This suggests that the presence of aluminol sites is a determining factor for adsorption and this provides an interesting leverage to study the correlation of flow reduction with adsorption. To do so, we introduced aluminol sites on a pure silica porous disc by prior treatment with $\mathrm{Al}_{13}$ polycations, $\left[\mathrm{Al}^{I V} \mathrm{O}_{4} \mathrm{Al} l_{12}^{V I}(\mathrm{OH})_{24}\left(\mathrm{H}_{2} \mathrm{O}\right)_{12}\right]^{7+}$. The aluminum treatment consists in putting in contact duing about $12 \mathrm{~h}$ the silica materials with a solution of $\mathrm{Al}_{13}$ polycations which was known to create aluminosilicate sites on the surface of silica particles. ${ }^{40}$ Titration of the $\mathrm{Al}_{13}$ solution with $\mathrm{NaOH}(0.1 \mathrm{M})$ before and after contact with silica particles allowed determining the concentration of aluminum adsorbed at the surface of particles during the treatment. On the porous disks used for injectivity loss measurements (vide infra), after crushing, an adsorbed amount of $1.3510^{-1} \mathrm{mg}$ of aluminum per grams of solids was measured. It decreased to $8.7010^{-2} \mathrm{mg} / \mathrm{g}$ for the not crushed silica porous disc due to its lower specific area and possibly diffusional limitations. Indeed the specific areas of those two materials are too weak to be measured but one can assume that the porous disc developed a higher specific area when crushed explaining its ability to contain more aluminum at its surface.

To confirm that the aluminum treatment introduced aluminol sites on the surface of the porous disc, NMR ${ }^{27} \mathrm{Al}$ experiments were conducted on crushed silica porous discs before and after aluminum treatment. While the pristine sample is devoid of aluminum and thus provided no NMR signal, a significant resonance around 0 ppm corresponding to aluminum in octahedral coordination was detected after treatment, thereby confirming the formation of aluminol sites. Adsorption isotherms confirmed that hydrolysed polyacrylamide did not adsorb significantly on the pure silica disc material but that the treatment with aluminum strongly promoted the adsorption of polyacrylamide (Figure 7) in agreement with the literature. ${ }^{65}$ 


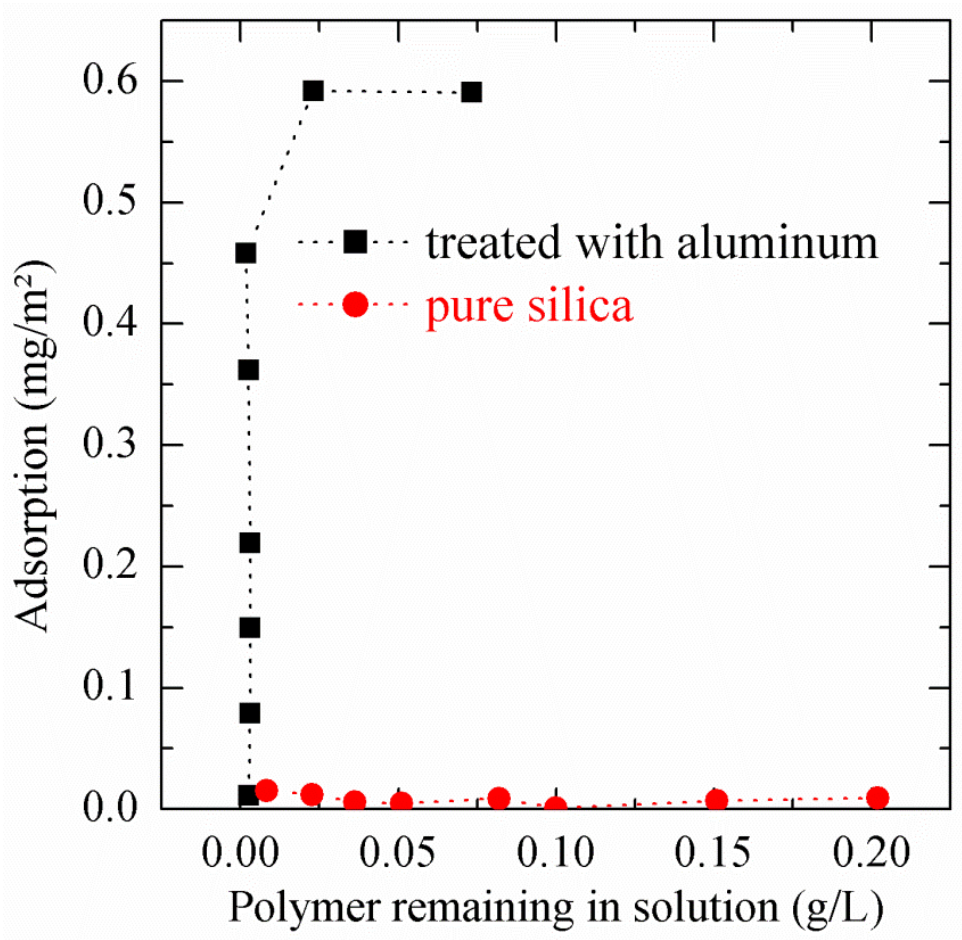

Fig. 7. Adsorption of HPAM-10 on crushed silica porous discs at $30 \mathrm{wt} \%$ with or without aluminum pretreatment at $\mathrm{I}=0.15 \mathrm{M}$ in $\mathrm{CaCl}_{2}$.

Considering that adsorption takes place predominantly on aluminol sites, comparing the flow of water through silica porous discs contacted with polymer, with or without prior aluminium treatment provided a means to clearly evidence the relationship between adsorption and permeability reduction.

Indeed, a f-PAM solution at a polymer concentration of $800 \mathrm{ppm}$ was injected on porous silica discs without aluminum treatment. After polymer flow and $16 \mathrm{~h}$ at rest, the polymer solution present in pores was flushed with water. After complete washing, the pressure drop stabilized at a value higher than before the polymer injection, on the pristine porous silica disc. This increase of pressure resulted in a permeability reduction to water (RRF) of 1.4 after the injection of f-PAM (Table 5). Regarding the negligible adsorption measured on the porous discs, a value of RRF close to 1 was expected in the absence of retention. Indeed, as explained in the experimental part, an additional disc mounted upstream mostly prevents the retention effect in the disc of interest. On the contrary, the aluminum pre-treatment revealed a drastic effect since the permeability reduction raised to 120 , value two orders of magnitude more than without aluminum treatment. This result clearly demonstrated the efficiency of aluminum treatment to boost the adsorption capacity of non-adsorbing silica and consequently 
the strong link between PAM adsorption and the development of a hydrodynamic resistance in porous medium.

\section{Table 5}

Permeability reduction to water measured after the injection of a solution of commercial polyacrylamide (f-PAM, Flopaam 3630S) through silica porous discs with or without aluminum treatment. $K_{\mathrm{w}}$ is the initial water permeability, $\Phi$ is the porosity and RRF is the permeability reduction. Another untreated disk is mounted upstream to factor out retention effects.

\begin{tabular}{cccc}
\hline & \multicolumn{3}{c}{ Porous medium } \\
& $\mathrm{Kw}(\mathrm{mD})$ & $\Phi(\%)$ & $\mathrm{RRF}$ \\
\hline $\begin{array}{c}\text { Without aluminum } \\
\text { treatment } \\
\text { With aluminum } \\
\text { treatment }\end{array}$ & 810 & 46 & 1.4 \\
\hline
\end{tabular}

\section{Conclusion}

In the present work, we clearly demonstrated the strong correlation between the adsorption of acrylamide-based polymers, PAM/HPAM, on siliceous materials and the loss of injectivity observed in the case of water injection into silica porous materials. First, the literature showed that there was a lack of clarity concerning the affinity of acrylamide-based polymers for silica surface. $^{22-38,45}$ In order to clarify the understanding about the polymer adsorption on silica surface and the key parameters acting on this mechanism, we have built a preliminary critical review of the literature concerning the possible specific interactions between acrylamide polymers and silica surface. This review revealed contrasting and sometimes difficult to reconcile results. This literature review was consequently supplemented with relevant polymer adsorption experiments on various siliceous materials with well-defined acrylamidebased polymers, i.e. linear neutral PAM and statistical copolymers P(AM-st-AA) synthesized by RAFT polymerization and assimilated to HPAM. The use of these polymers with 
controlled average molar mass, molar mass distributions and chemical composition together with a detailed characterization of the siliceous materials allowed us to bring to light the parameters critical to polymer adsorption (nature of the chemical groups onto the silica surface, the polymer anionicity, the $\mathrm{pH}$ of the solution, the ionic strength ...). It appeared clearly that aluminol groups present onto the siliceous materials even as trace contaminants were mainly responsible to the polymer adsorption observed in our present work.

Then, we correlate our static experiments relating to polymer adsorption experiments to dynamic experiments where water was injected in porous materials previously put in contact with polymer solution. The observed loss of injectivity can have many causes such hydrodynamic retention, mechanical entrapment or adsorption. We clearly showed that polymer adsorption is the leading cause for the loss of injectivity of orders of magnitudes sometimes observed by oil industry. This result contrasts with reported works where the authors directly concluded to polymer adsorption using dynamic experiments. ${ }^{3,4}$

Finally, this works contains both fundamental concepts and industrial concerns which allows, at least in the case of PAM/HPAM silica system, to have a clear vision on the consequence of the adsorption on the flow in porous material.

\section{Acknowledgments}

We gratefully acknowledge Total for financial support. 


\section{References}

1. G. Chauveteau, Fundamental Criteria in Polymer Flow through Porous Media and their Importance in the Performance Differences of Mobility-Control Buffers Advances in Chemistry Series (1986), 227-267.

2. P. L. J. Zitha, G. Chauveteau, L. Leger, Unsteady-state flow of flexible polymers in porous media, Journal of colloid and interface science 234 (2001), 269-283.

3. A. Zaitoun, N. Kohler, in: SPE International Symposium on Oilfield Chemistry, Society of Petroleum Engineers: San Antonio, Texas, 1987.

4. A. Zaitoun, N. Kohler, in: SPE Annual Technical Conference and Exhibition, Society of Petroleum Engineers: Houston, Texas, 1988.

5. M. Ben-Hur, M. Malik, J. Letey, U. Mingelgrin, Adsorption of polymers on clays as affected by clay charge and structure, polymer properties, and water quality, Soil Science 153 (1992), 349-356.

6. D. Fox, R. B. Bryan, Influence of a polyacrylamide soil conditioner on runoff generation and soil erosion: Field tests in Baringo District, Kenya, Soil Technology 5 (1992), 101-119.

7. B. Janczuk, T. Bialopiotrowicz, A. Kliszcz, B. Bilinski, J. Stawinski, Influence of polyacrylamide on the surface free energy and wettability of a chernozem soil, Geoderma 50 (1991), 173-184.

8. R. D. Lentz, R. E. Sojka, Field results using polyacrylamide to manage furrow erosion and infiltration, Soil Science 158 (1994), 274-282.

9. J. Letey, Adsorption and desorption of polymers on soil, Soil Science 158 (1994), 244-248.

10. A. R. Mitchell, Polyacrylamide application in irrigation water to increase infiltration, Soil Science 141 (1986), 353-358.

11. A. Nadler, M. Magaritz, L. Leib, PAM application techniques and mobility in soil, Soil Science 158 (1994), 249-254.

12. A. Nadler, E. Perfect, B. D. Kay, Effect of polyacrylamide application on the stability of dry and wet aggregates, Soil Science Society of America Journal 60 (1996), 555-561.

13. I. Shainberg, G. J. Levy, P. Rengasamy, H. Frenkel, Aggregate stability and seal formation as affected by drops'impact energy and soil amendments, Soil Science 154 (1992), 113-119.

14. R. E. Terry, S. D. Nelson, Effects of polyacrylamide and irrigation method on soil physical properties, Soil Science 141 (1986), 317-320.

15. X. C. Zhang, W. P. Miller, Polyacrylamide effect on infiltration and erosion in furrows, Soil Science Society of America Journal 60 (1996), 866-872.

16. H. Bessaies-Bey, R. Baumann, M. Schmitz, M. Radler, N. Roussel, Effect of polyacrylamide on rheology of fresh cement pastes, Cement and Concrete Research 76 (2015), 98-106. 
17. C. Negro, A. Blanco, E. Fuente, L. M. Sanchez, J. Tijero, Influence of flocculant molecular weight and anionic charge on flocculation behaviour and on the manufacture of fibre cement composites by the Hatschek process, Cement and Concrete Research 35 (2005), 2095-2103.

18. C. Negro, E. Fuente, L. M. Sanchez, A. Blanco, J. Tijero, Evaluation of an alternative flocculation system for manufacture of fiber-cement composites, Industrial and Engineering Chemistry Research 45 (2006), 6672-6678.

19. C. Negro, L. M. Sanchez, E. Fuente, A. Blanco, J. Tijero, Polyacrylamide induced flocculation of a cement suspension, Chemical Engineering Science 61 (2006), 2522-2532.

20. C. Negro, L. M. Sanchez, H. Fuente, A. Blanco, Effects of flocculants and sizing agents on bending strength of fiber cement composites, Cement and Concrete Research 35 (2005), 2104-2109.

21. D. A. Z. Wever, F. Picchioni, A. A. Broekhuis, Polymers for enhanced oil recovery: A paradigm for structure-property relationship in aqueous solution, Progress in Polymer Science 36 (2011), 1558-1628.

22. A. R. Al-Hashmi, P. F. Luckham, Characterization of the adsorption of high molecular weight non-ionic and cationic polyacrylamide on glass from aqueous solutions using modified atomic force microscopy, Colloids and Surfaces a-Physicochemical and Engineering Aspects 358 (2010), 142-148.

23. A. R. Al-Hashmi, P. F. Luckham, R. S. Al-Maamari, A. Zaitoun, H. H. Al-Sharji, The role of hydration degree of cations and anions on the adsorption of high-molecular-weight nonionic polyacrylamide on glass surfaces, Colloids and Surfaces a-Physicochemical and Engineering Aspects 415 (2012), 91-97.

24. A. R. Al-Hashmi, P. F. Luckham, J. Y. Y. Heng, R. S. Al-Maamari, A. Zaitoun, H. H. AlSharji, T. K. Al-Wehaibi, Adsorption of High-Molecular-Weight EOR Polymers on Glass Surfaces Using AFM and QCM-D, Energy \& Fuels 27 (2013), 2437-2444.

25. M. Bjelopavlic, H. El-Shall, B. M. Moudgil, in: Polymers in Particulate Systems: Properties and Applications, V. A. Hackley; P. Somasundaran; J. A. Lewis, (Eds.) New York, Vol. 104, pp 105133.

26. C. A. Grattoni, P. F. Luckham, X. D. Jing, L. Norman, R. W. Zimmerman, Polymers as relative permeability modifiers: adsorption and the dynamic formation of thick polyacrylamide layers, Journal of Petroleum Science and Engineering 45 (2004), 233-245.

27. G. J. Graveling, K. V. Ragnarsdottir, G. C. Allen, J. Eastman, P. V. Brady, S. D. Balsley, D. R. Skuse, Controls on polyacrylamide adsorption to quartz, kaolinite, and feldspar, Geochimica et cosmochimica acta 61 (1997), 3515-3523.

28. O. Griot, Kitchene.Ja, Role of Surface Silanol Groups in Flocculation of Silica Supsensions by Polyacrylamide .2. Surface Changes of Silica Suspensions on Ageing Transactions of the Faraday Society 61 (1965), 1032-1038.

29. O. Griot, J. A. Kitchener, Ageing of Silica Suspensions in Water and its Influence on Flocculation by Polyacrylamide, Nature 200 (1963), 1004-1005. 
30. O. Griot, J. A. Kitchener, Role of Surface Silanol Groups in Flocculation of Silica Supsensions by Polyacrylamide .1. Chemistry of Adsorption Process Transactions of the Faraday Society 61 (1965), 1026-1031.

31. Y. Guevellou, C. Noik, J. Lecourtier, D. Defives, Polyacrylamide Adsorption onto Dissolving Minerals at Basic pH, Colloids and Surfaces a-Physicochemical and Engineering Aspects 100 (1995), 173-185.

32. J. Lecourtier, L. T. Lee, G. Chauveteau, Adsorption of polyacrylamides on siliceous minerals, Colloids and Surfaces 47 (1990), 219-231.

33. L.-T. Lee, P. Somasundaran, Adsorption of polyacrylamide on oxide minerals, Langmuir 5 (1989), 854-860.

34. J. H. Lu, L. Wu, J. Letey, Effects of soil and water properties on anionic polyacrylamide sorption, Soil Science Society of America Journal 66 (2002), 578-584.

35. M. Page, J. Lecourtier, C. Noik, A. Foissy, Adsorption of Polyacrylamides and Polysaccharides on Siliceous Materials and Kaolinite - Influence of Temperature Journal of colloid and interface science 161 (1993), 450-454.

36. Y. Samoshina, A. Diaz, Y. Becker, T. Nylander, B. Lindman, Adsorption of cationic, anionic and hydrophobically modified polyacrylamides on silica surfaces, Colloids and Surfaces aPhysicochemical and Engineering Aspects 231 (2003), 195-205.

37. P. Somasundaran, L. T. Lee, Polymer-Surfactant Interactions in Flotation of Quartz, Separation Science and Technology 16 (1981), 1475-1490.

38. M. Wisniewska, The temperature effect on the adsorption mechanism of polyacrylamide on the silica surface and its stability, Applied Surface Science 258 (2012), 3094-3101.

39. C. Gérardin, N. Sanson, F. Bouyer, F. Fajula, J.-L. Putaux, M. Joanicot, T. Chopin, Highly Stable Metal Hydrous Oxide Colloids by Inorganic Polycondensation in Suspension, Angewandte Chemie International Edition 42 (2003), 3681-3685.

40. C. Parneix, J. Persello, R. Schweins, B. Cabane, How Do Colloidal Aggregates Yield to Compressive Stress?, Langmuir 25 (2009), 4692-4707.

41. E. Read, A. Guinaudeau, D. J. Wilson, A. Cadix, F. Violleau, M. Destarac, Low temperature RAFT/MADIX gel polymerisation: access to controlled ultra-high molar mass polyacrylamides, Polymer Chemistry 5 (2014), 2202-2207.

42. M. Wisniewska, The structure of electrical double layer of silica in the presence of polyvinyl alcohol (PVA) at different temperatures, Materials Letters 64 (2010), 1611-1613.

43. F. Lafuma, K. Wong, B. Cabane, Bridging of Colloidal Particles Through Adsorbed polymers, Journal of colloid and interface science 143 (1991), 9-21.

44. R. S. Parnas, M. Chaimberg, V. Taepaisitphongse, Y. Cohen, The adsorption of Polyvinylpyrrolidone and Polyethylene Oxide onto Chemically Modified Silica, Journal of colloid and interface science 129 (1989), 441-450. 
45. H. Wan, R. S. Seright, Is Polymer Retention Different Under Anaerobic vs. Aerobic Conditions?, Spe Journal 22 (2016), 431-437.

46. I. S. Chuang, D. R. Kinney, C. E. Bronnimann, R. C. Zeigler, G. E. Maciel, Effects of protonproton spin exchange in the silicon-29 CP-MAS NMR spectra of the silica surface, The Journal of Physical Chemistry 96 (1992), 4027-4034.

47. S. Leonardelli, L. Facchini, C. Fretigny, P. Tougne, A. P. Legrand, Si-29 NMR study of silica, Journal of the American Chemical Society 114 (1992), 6412-6418.

48. G. E. Maciel, C. E. Bronnimann, R. C. Zeigler, I.-S. Chuang, D. R. Kinney, E. A. Keiter, in: ACS Advances in Chemistry Series, H. E. Bergna, (Ed.) 1994; Vol. 234, pp 269-286.

49. B. Pfleiderer, K. Albert, E. Bayer, L. Van de Ven, J. De Haan, C. A. M. G. Cramers, A new approach to the silica gel surface: characterization of different surface regions by silicon-29 magic angle spinning NMR relaxation parameters and consequences for quantification of silica gels by NMR, Journal of Physical Chemistry 94 (1990), 4189-4194.

50. A. Burneau, O. Barres, J. P. Gallas, J. C. Lavalley, Comparative study of the surface hydroxyl groups of fumed and precipitated silicas. 2. Characterization by infrared spectroscopy of the interactions with water, Langmuir 6 (1990), 1364-1372.

51. J. J. Fripiat, J. Uytterhoeven, Hydroxyl content in silica gel "Aerosil"可, The Journal of Physical Chemistry 66 (1962), 800-805.

52. J. P. Gallas, J. C. Lavalley, A. Burneau, O. Barres, Comparative study of the surface hydroxyl groups of fumed and precipitated silicas. 4. Infrared study of dehydroxylation by thermal treatments, Langmuir 7 (1991), 1235-1240.

53. A. P. Legrand, H. Hommel, A. Tuel, A. Vidal, H. Balard, E. Papirer, P. Levitz, M. Czernichowski, R. Erre, H. Vandamme, J. P. Gallas, J. F. Hemidy, J. C. Lavalley, O. Barres, A. Burneau, Y. Grillet, Hydroxyls of silica powders, Advances in Colloid and Interface Science 33 (1990), 91-330.

54. J. B. Peri, Infrared study of $\mathrm{OH}$ and $\mathrm{NH} 2$ groups on the surface of a dry silica aerogel, The Journal of Physical Chemistry 70 (1966), 2937-2945.

55. A. J. Van Roosmalen, J. C. Mol, An infrared study of the silica gel surface. 1. Dry silica gel, The Journal of Physical Chemistry 82 (1978), 2748-2751.

56. L. T. Zhuravlev, The surface chemistry of amorphous silica. Zhuravlev model, Colloids and Surfaces A: Physicochemical and Engineering Aspects 173 (2000), 1-38.

57. A. Tuel, H. Hommel, A. P. Legrand, Y. Chevallier, J. C. Morawski, Solid state NMR studies of precipitated and pyrogenic silicas, Colloids and surfaces 45 (1990), 413-426.

58. L. T. Zhuravlev, Concentration of hydroxyl groups on the surface of amorphous silicas, Langmuir 3 (1987), 316-318.

59. G. J. Young, Interaction of water vapor with silica surfaces, Journal of Colloid Science 13 (1958), 67-85. 
60. V. Dugas, Y. Chevalier, Surface hydroxylation and silane grafting on fumed and thermal silica, Journal of colloid and interface science 264 (2003), 354-361.

61. A. A. Agzamkhodzhaev, L. T. Zhuravlev, A. V. Kiselev, K. Y. Shengeliya, in: 1974; pp 10361039.

62. D. W. Sindorf, G. E. Maciel, Silicon-29 NMR study of dehydrated/rehydrated silica gel using cross polarization and magic-angle spinning, Journal of the American Chemical Society 105 (1983), 1487-1493.

63. E. Pefferkorn, L. Nabzar, A. Carroy, Adsorption of polyacrylamide to Na kaolinite: Correlation between clay structure and surface properties, Journal of colloid and interface science 106 (1985), 94-103.

64. E. Pefferkorn, Structure and stability of natural organic matter/soil complexes and related synthetic and mixed analogues, Advances in colloid and interface science 73 (1997), 127-200.

65. E. Pefferkorn, Polyacrylamide at solid/liquid interfaces, Journal of colloid and interface science 216 (1999), 197-220.

66. N. K. Maurya, A. Mandal, Studies on behavior of suspension of silica nanoparticle in aqueous polyacrylamide solution for application in enhanced oil recovery, Petroleum Science and Technology 34 (2016), 429-436.

67. V. Adibnia, K. W. Cho, R. J. Hill, Nanoparticle Coupling to Hydrogel Networks: New Insights from Electroacoustic Spectroscopy, Macromolecules 50 (2017), 4030-4038.

68. V. Adibnia, R. J. Hill, Viscoelasticity of near-critical silica-polyacrylamide hydrogel nanocomposites, Polymer 112 (2017), 457-465.

69. Z. Hu, M. Haruna, H. Gao, E. Nourafkan, D. Wen, Rheological Properties of Partially Hydrolyzed Polyacrylamide Seeded by Nanoparticles, Industrial \& Engineering Chemistry Research 56 (2017), 3456-3463.

70. M. Lotfollahi, R. Farajzadeh, M. Delshad, A. Al-Abri, B. M. Wassing, R. Al-Mjeni, K. Awan, P. Bedrikovetsky, Mechanistic Simulation of Polymer Injectivity in Field Tests, Spe Journal 21 (2016), 1178-1191.

71. P. L. J. Zitha, Kinetics of the bridging adsorption of flexible chains in porous media, Comptes Rendus De L Academie Des Sciences Serie Iv Physique Astrophysique 2 (2001), 787-796. 